\title{
Flat pairing and generalized Cheeger-Simons characters
}

\author{
Fabio Ferrari Ruffino ${ }^{1}$
}

Received: 9 September 2014 / Accepted: 27 November 2015 / Published online: 18 December 2015 (C) Tbilisi Centre for Mathematical Sciences 2015

\begin{abstract}
Let $h^{\bullet}$ be a multiplicative cohomology theory, $h_{\bullet}$ its dual homology theory and $\hat{h}^{\bullet}$ a differential refinement. We first construct the natural pairing between $h_{\bullet}$ and the flat part of $\hat{h}^{\bullet}$, generalizing the holonomy of a flat Deligne cohomology class. Then, in order to generalize the holonomy of any Deligne cohomology class, we define the generalized Cheeger-Simons characters. The latter are functions from suitably defined differential cycles to the cohomology ring of the point, such that the value on a trivial cycle only depends on the curvature.
\end{abstract}

Keywords Generalized cohomology theories · differential cohomology ·

Cheeger-Simons characters

\section{Introduction}

Let us consider the ordinary differential cohomology $\hat{H}^{\bullet}$ on a smooth manifold $X$ [8]. The group $\hat{H}^{n}(X)$ is canonically isomorphic to the group of the Cheeger-Simons differential characters of degree $n$. An element of the latter is a couple $(\chi, \omega)$, where $\chi$ is an $\mathbb{R} / \mathbb{Z}$-valued group morphism defined on the smooth $(n-1)$-cycles of $X$ (whose exponential is the holonomy), and $\omega$ is an integral $n$-form on $X$ (the curvature) such that, on a $p$-boundary $\partial D$, one has:

Communicated by Thomas Schick.

I would like to thank the referee for many helpful suggestions, critics and remarks, that changed the structure of the paper. I was supported by FAPESP (Fundação de Amparo à Pesquisa do Estado de São Paulo), processo 2014/03721-3, and I am thankful to the ICMC-USP, São Carlos, for its support during my staying there.

$凶 \quad$ Fabio Ferrari Ruffino ferrariruffino@gmail.com

1 Departamento de Matemática, Universidade Federal de São Carlos, Rod. Washington Luís, Km 235, C.P. 676, 13565-905 São Carlos, SP, Brazil 


$$
\chi(\partial D)=\int_{D} \omega \bmod \mathbb{Z} .
$$

In particular, if the class is flat, the holonomy only depends on the homology class of the cycle, and actually the flat part of $\hat{H}^{n}(X)$ is canonically isomorphic to $H^{n-1}(X ; \mathbb{R} / \mathbb{Z})$.

The aim of the present paper is to generalize this picture to a differential refinement $\hat{h}^{\bullet}$ of any multiplicative cohomology theory $h^{\bullet}[3,8,16]$. We start considering the flat case: a differential class of degree $n$ provides a morphism from the homology theory $h_{\bullet}$ to the cohomology ring of the point; we thus get a pairing between $h_{\bullet}$ and the flat part of $\hat{h}^{\bullet}$. Then, in order to consider even non-flat classes, we consider the geometrical definition of the homology theory $h_{\bullet}$ dual to $h^{\bullet}$, as described in [9], and we define a variant of that construction, using a suitable differential extension of cycles and boundaries. In this way it is possible to define the generalized Cheeger-Simons characters as functions from the differential cycles to the cohomology ring of the point, such that the value on a trivial cycle only depends on the curvature. In the case of $K$-theory this construction is equivalent to the one defined in [2].

The paper is organized as follows. In Sects. 2 and 3 we recall the preliminaries about differential cohomology and the corresponding notions of orientation and integration. In Sect. 4 we recall the notion of dual homology theory and its basic properties. In Sect. 5 we show the pairing between $h$. and the flat part of $\hat{h}^{\bullet}$. Finally, in Sect. 6 we construct a model of the homology groups via differential cycles and we define the generalized Cheeger-Simons characters.

\section{Differential cohomology}

Let $\mathcal{M}$ be the category of smooth manifolds or of smooth compact manifolds (even with boundary), and let $\mathcal{A}_{\mathbb{Z}}$ be the category of $\mathbb{Z}$-graded abelian groups. We consider a cohomology theory $h^{\bullet}$, defined on a category including $\mathcal{M}$. We use the following notation:

$$
\mathfrak{h}^{\bullet}:=h^{\bullet}(\{p t\}) \quad \mathfrak{h}_{\mathbb{R}}^{\bullet}:=\mathfrak{h}^{\bullet} \otimes_{\mathbb{Z}} \mathbb{R}
$$

Moreover, for any object $M$ of $\mathcal{M}$, we call ch $: h^{\bullet}(M) \rightarrow H_{\mathrm{dR}}^{\bullet}\left(M ; \mathfrak{h}_{\mathbb{R}}^{\bullet}\right)$ the generalized Chern character [8, sec. 4.8, p. 47]. In this introductory section, we follow [4, sec. 1].

Definition 2.1 A differential extension of $h^{\bullet}$ is a functor $\hat{h}^{\bullet}: \mathcal{M}^{\text {op }} \rightarrow \mathcal{A}_{\mathbb{Z}}$, together with the following natural transformations of $\mathcal{A}_{\mathbb{Z}}$-valued functors:

- $I: \hat{h}^{\bullet}(M) \rightarrow h^{\bullet}(M)$;

- $R: \hat{h}^{\bullet}(M) \rightarrow \Omega_{\mathrm{cl}}^{\bullet}\left(M ; \mathfrak{h}_{\mathbb{R}}^{\bullet}\right)$, called curvature;

- $a: \Omega^{\bullet-1}\left(M ; \mathfrak{h} \mathfrak{R}^{\bullet}\right) / \operatorname{Im}(d) \rightarrow \hat{h}^{\bullet}(M)$,

such that:

- $R \circ a=d$; 
- the following diagram is commutative:

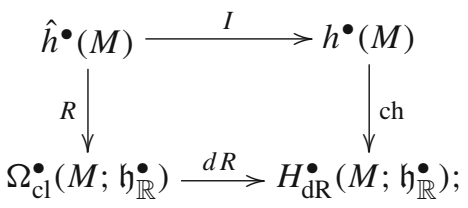

- the following sequence is exact:

$$
\hat{h}^{\bullet-1}(M) \stackrel{\text { ch }}{\longrightarrow} \Omega^{\bullet-1}\left(M ; \mathfrak{h}_{\mathbb{R}}^{\bullet}\right) / \operatorname{Im}(d) \stackrel{a}{\longrightarrow} \hat{h}^{\bullet}(M) \stackrel{I}{\longrightarrow} h^{\bullet}(M) \longrightarrow 0 .
$$

We also call $\hat{h}^{\bullet}$ differential cohomology theory.

A class $\hat{\alpha} \in \hat{h}^{n}(M)$ is called flat if and only if $R(\hat{\alpha})=0$. Considering flat classes, we get the functor $\hat{h}_{\mathrm{fl}}^{\bullet}: \mathcal{M} \rightarrow \mathcal{A}_{\mathbb{Z}}$. Thus, we get the following commutative hexagon:

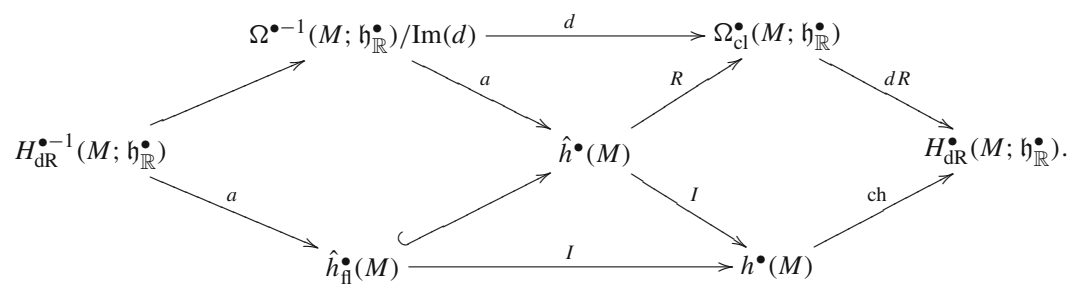

The following lemma easily follows from the previous axioms.

Lemma 2.2 (Homotopy formula) If $\hat{\alpha} \in \hat{h}^{\bullet}(I \times X)$ and $i_{0}, i_{1}: X \rightarrow I \times X$ are the natural embeddings, we have:

$$
i_{1}^{*} \hat{\alpha}-i_{0}^{*} \hat{\alpha}=a\left(\int_{I} R(\hat{\alpha})\right) .
$$

We now introduce integration. Given a functor $\mathcal{F}: \mathcal{M} \rightarrow \mathcal{C}$, for any category $\mathcal{C}$, we define the functor $S \mathcal{F}: \mathcal{M} \rightarrow \mathcal{C}$ defined by $S \mathcal{F}(M):=\mathcal{F}\left(S^{1} \times M\right)$ on objects and $S \mathcal{F}(f):=\mathcal{F}\left(\operatorname{id}_{S^{1}} \times f\right)$ on morphisms.

Definition 2.3 A differential extension with integration of $h^{\bullet}$ is a differential extension $\left(\hat{h}^{\bullet}, I, R, a\right)$ together with a natural transformation:

$$
\int_{S^{1}}: S \hat{h}^{\bullet+1} \rightarrow \hat{h}^{\bullet}
$$

such that:

- $\int_{S^{1}} \circ(t \times \mathrm{id})^{*}=-\int_{S^{1}}$, where $t: S^{1} \rightarrow S^{1}$ is defined by $t\left(e^{i \theta}\right):=e^{-i \theta}$;

- $\int_{S^{1}} \circ p^{*}=0$, where $p: S^{1} \times X \rightarrow X$ is the projection; 
- the following diagram is commutative:

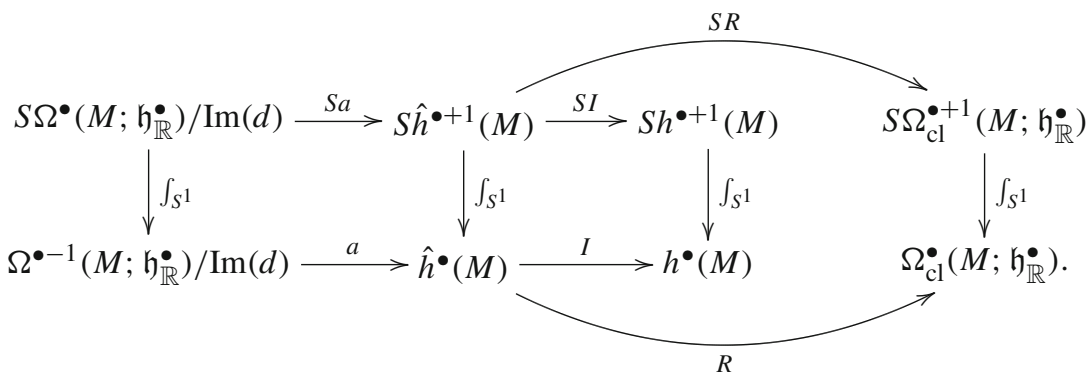

Let us consider a differential extension with integration $\left(\hat{h}^{\bullet}, I, R, a, \int_{S^{1}}\right)$. It is shown in [4, pp. 27-32] that, if $h^{\bullet}$ is rationally even (i.e., $\mathfrak{h}_{\mathbb{R}}^{2 k+1}=0$ for every $k \in \mathbb{Z}$ ), $\mathfrak{h}^{k}$ is countably generated for every $k \in \mathbb{Z}$ and $\mathcal{M}$ is the category of all smooth manifolds, there is an isomorphism of functors $\hat{h}_{\mathrm{fl}}^{\bullet} \simeq h^{\bullet-1}(\cdot ; \mathbb{R} / \mathbb{Z})$. If $\mathcal{M}$ is the category of compact manifolds, we must require that $\mathfrak{h}^{2 k+1}=0$ and $\mathfrak{h}^{2 k}$ is finitely generated for every $k \in \mathbb{Z}$. In these cases, the commutative hexagon (1) becomes:

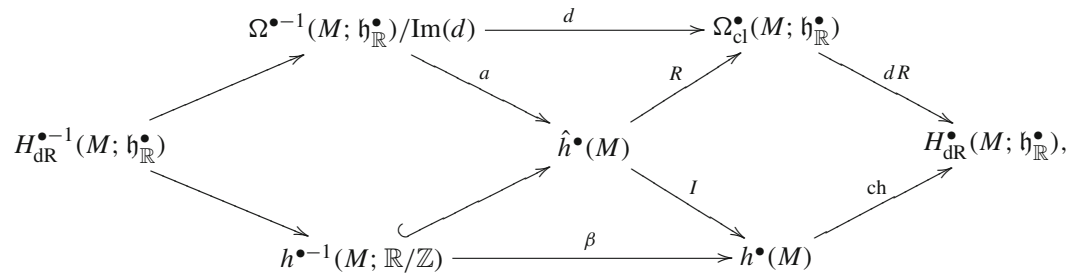

where $\beta$, in the last line, is the Bockstein map of the long exact sequence induced by the coefficient sequence $0 \rightarrow \mathbb{Z} \rightarrow \mathbb{R} \rightarrow \mathbb{R} / \mathbb{Z} \rightarrow 0$.

Finally, we introduce products, thus we suppose that $h^{\bullet}$ is a multiplicative cohomology theory. We call $\mathcal{R}_{\mathbb{Z}}$ the category of $\mathbb{Z}$-graded commutative rings. There is a natural forgetful functor $\mathcal{R}_{\mathbb{Z}} \rightarrow \mathcal{A}_{\mathbb{Z}}$, that we apply when needed, without writing it explicitly.

Definition 2.4 A multiplicative differential extension of $h^{\bullet}$ is a differential extension $\left(\hat{h}^{\bullet}, I, R, a\right)$ such that $\hat{h}^{\bullet}: \mathcal{M}^{\text {op }} \rightarrow \mathcal{R}_{\mathbb{Z}}$ and:

- $I$ and $R$ are multiplicative;

- $\hat{\alpha} \cdot a(\omega)=a(R(\hat{\alpha}) \wedge \omega)$ for every $\hat{\alpha} \in \hat{h}^{\bullet}(M)$ and $\omega \in \Omega^{\bullet}\left(M ; \mathfrak{h}_{\mathbb{R}}^{\bullet}\right) / \operatorname{Im}(d)$.

In the following we consider multiplicative differential extensions with integration.

\section{Orientation and integration}

Following [3, sec. 4.8-4.10], we briefly recall the topological notions of orientation and integration and we extend them to the differential case. 


\subsection{Topological orientation and integration}

Let $X$ be a compact manifold and $E \rightarrow X$ a real vector bundle of rank $n$. We call $E_{0}$ the fiber sub-bundle of $E$, containing the complement of the unit ball of each fiber of $E$, with respect to any fixed metric. The bundle $E$ is orientable with respect to a multiplicative cohomology theory $h^{\bullet}$ if there exists a Thom class $u \in h^{n}\left(E, E_{0}\right)[14$, p. 253], i.e. a class such that, for every $x \in X$, the restriction $\left.u\right|_{\left(E_{x}, E_{0, x}\right)}$ maps to \pm 1 under the isomorphism:

$$
h^{n}\left(E_{x}, E_{0, x}\right) \simeq h^{n}\left(\mathbb{R}^{n}, \mathbb{R}^{n} \backslash B^{n}\right) \simeq \tilde{h}^{n}\left(S^{n}\right) \simeq \mathfrak{h}^{0}
$$

Using the product $h^{\bullet}\left(E, E_{0}\right) \otimes_{\mathbb{Z}} h^{\bullet}(E) \rightarrow h^{\bullet}\left(E, E_{0}\right)$, we define the Thom isomorphism $\alpha \mapsto u \cdot \pi^{*} \alpha$, between $h^{\bullet}(X)$ and $h^{\bullet+n}\left(E, E_{0}\right)$. Thus, we define the integration map $\int_{E / X}: h^{\bullet}\left(E, E_{0}\right) \rightarrow h^{\bullet-n}(X)$ by $u \cdot \pi^{*} \alpha \mapsto \alpha$. If the characteristic of $\mathfrak{h}^{\bullet}$ is different from 2, the $n$-degree component of ch $u$ defines an orientation of $E$ in the usual sense, hence it is possible to integrate a compactly-supported form fiber-wise. We define the Todd class $\operatorname{Td}(u):=\int_{E / X} \operatorname{ch} u \in H_{\mathrm{dR}}^{0}\left(X ; \mathfrak{h}_{\mathbb{R}}^{\bullet}\right)$. The following formula holds:

$$
\int_{E / X} \operatorname{ch} \alpha=\operatorname{Td}(u) \cdot\left(\operatorname{ch} \int_{E / X} \alpha\right) .
$$

Lemma $3.1\left(2 \times 3\right.$ principle) Given two bundles $E, F \rightarrow X$, with projections $p_{E}$ : $E \oplus F \rightarrow E$ and $p_{F}: E \oplus F \rightarrow F$, we consider a triple $(u, v, w)$ of Thom classes on $E, F$ and $E \oplus F$ respectively, such that $w=p_{E}^{*} u \cdot p_{F}^{*} v$. Two elements of such a triple uniquely determine the third one.

For the proof see [14, prop. 1.10, p. 307]. In order to define the Gysin map associated to a smooth neat ${ }^{1}$ map of compact manifolds $f: Y \rightarrow X$, we consider a neat proper embedding $\iota: Y \hookrightarrow X \times \mathbb{R}^{N}$, such that $\pi_{X} \circ \iota=f$, and we endow the normal bundle $N_{\iota(Y)}\left(X \times \mathbb{R}^{N}\right)$ with a Thom class $u$. Then, we consider a neat tubular neighborhood $U$ of $\iota(Y)$ in $X \times \mathbb{R}^{N}$, a diffeomorphism $\varphi: N_{\iota(Y)}\left(X \times \mathbb{R}^{N}\right) \rightarrow U$ and the natural inclusion $i: U \rightarrow X$, inducing a push-forward in compactly-supported cohomology. The Gysin map $f_{!}: h^{\bullet}(Y) \rightarrow h^{\bullet-n}(X)$, for $n=\operatorname{dim} Y-\operatorname{dim} X$, is defined as:

$$
f_{!}(\alpha)=\int_{\mathbb{R}^{N}} i_{*} \varphi_{*}\left(u \cdot \pi^{*} \alpha\right) .
$$

We used the fact that, since $X$ is compact, we have $h^{\bullet}\left(E, E_{0}\right) \simeq h_{\mathrm{cpt}}^{\bullet}(E)$. The integration map is defined as follows: since $h_{\mathrm{cpt}}^{\bullet}\left(X \times \mathbb{R}^{N}\right)=\tilde{h}^{\bullet}((X \times$ $\left.\left.\mathbb{R}^{N}\right)^{+}\right) \simeq \tilde{h}^{\bullet}\left(\Sigma^{N}\left(X_{+}\right)\right)$, for $X_{+}=X \sqcup\{\infty\}$, we apply the suspension isomorphism $\tilde{h}^{\bullet}\left(\Sigma^{N}\left(X_{+}\right)\right) \simeq \tilde{h}^{\bullet-n}\left(X_{+}\right) \simeq h^{\bullet-n}(X)$. This construction of the Gysin map naturally leads to the following definition.

\footnotetext{
${ }^{1}$ We recall that $f: Y \rightarrow X$ is neat if $f^{-1}(\partial X)=\partial Y$ and $d f_{y}: T_{y} Y / T_{y}(\partial Y) \rightarrow T_{f(x)} X / T_{f(x)}(\partial X)$ is an isomorphism for every $y \in \partial Y$ [8, Appendix C.2, p.90]. See also [11, pp. 27ff] about neat submanifolds and tubular neighborhoods.
} 
Definition 3.2 A representative of an $h^{\bullet}$-orientation of a smooth neat map between compact manifolds $f: Y \rightarrow X$ is the datum of:

- a neat embedding $\iota: Y \hookrightarrow X \times \mathbb{R}^{N}$, for any $N \in \mathbb{N}$, such that $\pi_{X} \circ \iota=f$;

- a Thom class $u$ of the normal bundle $N_{\iota(Y)}\left(X \times \mathbb{R}^{N}\right)$;

- a diffeomorphism $\varphi: N_{\iota(Y)}\left(X \times \mathbb{R}^{N}\right) \rightarrow U$, for $U$ a neat tubular neighborhood of $\iota(Y)$ in $X \times \mathbb{R}^{N}$.

Let us consider a vector $v_{y} \in N_{\iota(Y)}\left(X \times \mathbb{R}^{N}\right)_{\iota(y)}$. It is sent by $\varphi$, as defined in 3.2 , to a point $\varphi\left(v_{y}\right) \in X \times \mathbb{R}^{N}$. If $f$ is a submersion, we can require that the first component of $\varphi\left(v_{y}\right)$ is $f(y)$. This means that the following diagram commutes:

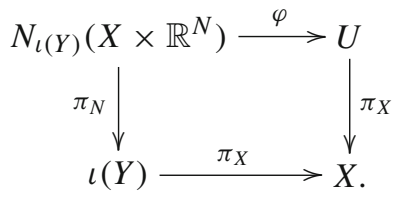

Definition 3.3 A representative of an $h^{\bullet}$-orientation of $f: Y \rightarrow X$ is proper if diagram (6) commutes.

Lemma 3.4 Let $f: Y \rightarrow X$ be a submersion. Then, for any neat embedding $\iota$ : $Y \hookrightarrow X \times \mathbb{R}^{N}$ and any Thom class $u$ of the normal bundle, there exists a proper representative $(\iota, u, \varphi)$ of an $h^{\bullet}$-orientation of $f$.

Proof We define the following projection:

$$
\begin{gathered}
p:\left.T\left(X \times \mathbb{R}^{N}\right)\right|_{\iota(Y)} \rightarrow\left(\iota(Y) \times \mathbb{R}^{N}\right) / d \iota(\operatorname{Ker} d f) \\
\left(d f_{y}(v), w\right) \mapsto\left[w-\pi_{\mathbb{R}^{N}}\left(d \iota_{y}(v)\right)\right] .
\end{gathered}
$$

It is easy to prove that $p$ is surjective and $\operatorname{Ker} p=T(\iota(Y))$, hence $N_{\iota(Y)}\left(X \times \mathbb{R}^{N}\right) \simeq$ $\left(\iota(Y) \times \mathbb{R}^{N}\right) / d \iota($ Ker $d f)$. Using the standard metric on $\mathbb{R}^{N}$, we can identify the quotient $\left(\iota(Y) \times \mathbb{R}^{N}\right) / d \iota(\operatorname{Ker} d f)$ with the sub-bundle $d \iota\left(\operatorname{Ker} d f_{y}\right)^{\perp}$, hence we can apply $\varphi$ in such a way that the fiber on $\iota(y)$ is sent to an open subset of $\{f(y)\} \times \mathbb{R}^{N}$ (the reader can work out the details).

We now introduce a suitable equivalence relation among representatives of orientations. First we need a generalization of Definition 3.3. Let us consider a representative $(J, U, \Phi)$ of an $h^{\bullet}$-orientation of id $\times f: I \times Y \rightarrow I \times X$ and a neighborhood $V \subset I$ of $\{0,1\}$. We say that the representative is proper on $V$ if a vector $(x, v)_{(t, y)} \in$ $N_{\iota(V \times Y)}\left(V \times X \times \mathbb{R}^{N}\right)_{\iota(t, y)}$ is sent by $\Phi$ to a point $\Phi\left((x, v)_{(t, y)}\right) \in V \times X \times \mathbb{R}^{N}$ whose first component is $t$. This means that the following diagram commutes:

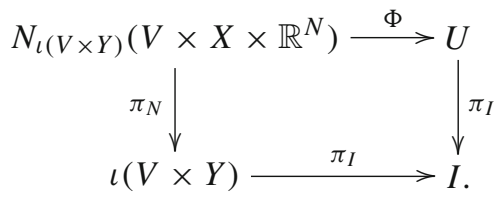


In this case, calling $f_{0}:=\operatorname{id}_{\{0\}} \times f$ and $f_{1}:=\operatorname{id}_{\{1\}} \times f$, we can define the restrictions $\left.(J, U, \Phi)\right|_{f_{0}}$ and $\left.(J, U, \Phi)\right|_{f_{1}}$.

Definition 3.5 A homotopy between two representatives $(\iota, u, \varphi)$ and $\left(\iota^{\prime}, u^{\prime}, \varphi^{\prime}\right)$ of an $h^{\bullet}$-orientation of $f: Y \rightarrow X$ is a representative $(J, U, \Phi)$ of an $h^{\bullet}$-orientation of id $\times f: I \times Y \rightarrow I \times X$, such that:

- $(J, U, \Phi)$ is proper over a neighborhood $V \subset I$ of $\{0,1\}$;

- $\left.(J, U, \Phi)\right|_{f_{0}}=(\iota, u, \varphi)$ e $\left.(J, U, \Phi)\right|_{f_{1}}=\left(\iota^{\prime}, u^{\prime}, \varphi^{\prime}\right)$.

On the trivial bundle $X \times \mathbb{R}^{N}$ there is a canonical Thom class, defined in the following way. On $p t \times \mathbb{R}^{N}$, whose compactification is $p t \times S^{N}$, we put the class $u_{0} \in \tilde{h}^{N}\left(S^{N}\right)$ corresponding to the suspension of $1 \in \mathfrak{h}^{0}$. Then, we put on $X \times \mathbb{R}^{N}$ the class $\pi_{\mathbb{R}^{N}}^{*} u_{0}$.

Definition 3.6 Let us consider a representative $(\iota, u, \varphi)$, with $\iota: Y \hookrightarrow X \times \mathbb{R}^{N}$.

- For any $L \in \mathbb{N}$, we define $\iota^{\prime}: Y \hookrightarrow X \times \mathbb{R}^{N+L}$ by $\iota^{\prime}(y):=(\iota(y), 0)$. Then $N_{\iota^{\prime}(Y)}\left(X \times \mathbb{R}^{N+L}\right) \simeq N_{\iota(Y)}\left(X \times \mathbb{R}^{N}\right) \oplus\left(\iota(Y) \times \mathbb{R}^{L}\right)$.

- We put the canonical orientation $u_{0}$ on the trivial bundle $\iota(Y) \times \mathbb{R}^{L}$, and the orientation $u^{\prime}$ induced by $u$ and $u_{0}$ on $N_{\iota^{\prime}(Y)}\left(X \times \mathbb{R}^{N+L}\right)$.

- Finally, for $v_{y} \in N_{\iota(Y)}\left(X \times \mathbb{R}^{N}\right)$ and $w \in \mathbb{R}^{L}$, we define $\varphi^{\prime}\left(v_{y}, w\right):=$ $\left(\varphi\left(v_{y}\right), w\right) \in X \times \mathbb{R}^{N+L}$.

The representative $\left(\iota^{\prime}, u^{\prime}, \varphi^{\prime}\right)$ is called equivalent by stabilization to $(\iota, u, \varphi)$.

Definition 3.7 A $\hat{h}^{\bullet}$-orientation on $f: Y \rightarrow X$ is an equivalence class $[\iota, u, \varphi]$ of representatives, up to the equivalence relation generated by homotopy and stabilization.

The Gysin map $f$ ! only depends on the $\hat{h}^{\bullet}$-orientation of $f$, not on the specific representative ([10, Theorem 5.24, p. 233], [3, sec. 4.9]). Moreover, because of the uniqueness up to homotopy and stabilization of the tubular neighborhood and of the diffeomorphism with the normal bundle, the class $[\iota, u, \varphi]$ does not depend on $\varphi$, hence we denote it by $[\iota, u]$.

Lemma 3.8 Given an $h^{\bullet}$-oriented map $f: Y \rightarrow X$, the Gysin map is a morphism of $\hat{h}^{\bullet}(X)$-modules, i.e., for any $\alpha \in h^{\bullet}(Y)$ and $\beta \in h^{\bullet}(X)$ :

$$
f_{!}\left(\alpha \cdot f^{*} \beta\right)=f_{!}(\alpha) \cdot \beta .
$$

For the proof see [10, Theorem 5.24, p. 233].

Definition 3.9 Let $f: Y \rightarrow X$ and $g: X \rightarrow W$ be $h^{\bullet}$-oriented maps, with orientations $[\iota, u]$ and $[\kappa, v]$, where $\iota: Y \hookrightarrow X \times \mathbb{R}^{N}$ and $\kappa: X \hookrightarrow W \times \mathbb{R}^{L}$. There is a naturally induced $h^{\bullet}$-orientation on $g \circ f: Y \rightarrow W$, that we denote by $[\kappa, v][\iota, u]$, defined in the following way:

- we choose the embedding $\xi=\left(\kappa, \operatorname{id}_{\mathbb{R}^{N}}\right) \circ \iota: Y \hookrightarrow W \times \mathbb{R}^{L+N}$; 
- on the normal bundle $N_{\xi(Y)}\left(W \times \mathbb{R}^{L+N}\right) \simeq N_{l(Y)}\left(X \times \mathbb{R}^{L}\right) \oplus N_{\kappa(X) \times \mathbb{R}^{L}}(W \times$ $\left.\mathbb{R}^{L+N}\right)\left.\left.\right|_{\xi(Y)} \simeq N_{l(Y)}\left(X \times \mathbb{R}^{L}\right) \oplus\left(\pi_{N}^{*} N_{\kappa(X)} W \times \mathbb{R}^{L}\right)\right|_{\xi(Y)}$, for $\pi_{L}: \mathbb{R}^{L+N} \rightarrow$ $\mathbb{R}^{N}$, we put the Thom class $w$ induced from the ones on $N_{l(Y)}\left(X \times \mathbb{R}^{L}\right)$ and $N_{\kappa(X)}\left(W \times \mathbb{R}^{N}\right)$.

We set $[\kappa, v][\iota, u]:=[\xi, w]$.

Lemma 3.10 With the data of Definition 3.9, $(g \circ f) !=g_{!} \circ f !$.

For the proof see [10, Theorem 5.24, p. 233]. The following lemma is a consequence of Lemma 3.1 and of the uniqueness up to homotopy and stabilization of the embedding l.

Lemma 3.11 $\left(2 \times 3\right.$ principle) Let $f: Y \rightarrow X$ and $g: X \rightarrow W$ be $h^{\bullet}$-oriented neat maps, with orientations $[\iota, u]$ and $[\kappa, v]$, and let $[\xi, w]:=[\kappa, v][\iota, u]$ be the orientation induced on $g \circ f$. Two elements of the triple $([\iota, u],[\kappa, v],[\xi, w])$ uniquely determine the third one.

Finally, we consider the orientation of manifolds.

Definition 3.12 An $h^{\bullet}$-orientation of a manifold without boundary $X$ is an $h^{\bullet}$ orientation of the map $p_{X}: X \rightarrow\{p t\}$.

By definition, giving an orientation to $p_{X}$ means fixing an orientation $u$ on the (stable) normal bundle of $X$. We set $\operatorname{Td}(X):=\operatorname{Td}(u)$. If $Y$ and $X$ are oriented, because of the $2 \times 3$ principle a map $f: Y \rightarrow X$ inherits an orientation, hence the Gysin map is well-defined.

Definition 3.13 An $h^{\bullet}$-orientation on a smooth manifold with boundary $X$ is the datum of:

- a neat map $\Phi: X \rightarrow I$ such that $\partial X=\Phi^{-1}\{0\}$;

- an $h^{\bullet}$-orientation of $\Phi$.

Again, giving an orientation to $\Phi$ means fixing an orientation $u$ on the stable normal bundle of $X$ : in fact, since $\Phi^{-1}\{1\}=\emptyset$, the normal bundle in $I \times \mathbb{R}^{N}$ is canonically isomorphic to the normal bundle in $[0,1) \times \mathbb{R}^{N} \simeq \mathbb{R}_{+}^{N+1}$, where $\mathbb{R}_{+}^{N}=\left\{\left(x_{1}, \ldots, x_{N}\right) \in \mathbb{R}^{N} \mid x_{N} \geq 0\right\}$. We set $\operatorname{Td}(X):=\operatorname{Td}(u)$.

An orientation $[\iota, u]$ on a manifold with boundary canonically induces an orientation $\left[\iota^{\prime}, u^{\prime}\right]$ on the boundary: in fact, calling $i_{\partial X}: \partial X \hookrightarrow X$ the natural embedding, we set $\iota^{\prime}:=\iota \circ i_{\partial X}: \partial X \hookrightarrow \mathbb{R}^{N-1}$ and $u^{\prime}:=\left.u\right|_{\partial X}$. The induced orientation does not depend on the representative chosen, since the tubular neighborhoods, the diffeomorphisms and the homotopies between them are neat, hence a homotopy between two representatives restricts to the boundary. The same holds for stabilization. Moreover, defining $\Phi: X \rightarrow I$ as in Definition 3.13, one has, for every $\alpha \in h^{\bullet}(X)$ :

$$
\left(p_{\partial X}\right) !\left(\left.\alpha\right|_{\partial X}\right)=\left.(\Phi ! \alpha)\right|_{\{0\}} .
$$

Such a formula is due to the fact that all the structures involved in the definition of the Gysin map for $p_{\partial X}$ are the restrictions to the boundary of the corresponding structures for $\Phi !$. 


\subsection{Differential orientation of a vector bundle}

If we consider a differential refinement $\hat{h}^{\bullet}$ of $h^{\bullet}$, in order to orient a vector bundle one just has to refine a Thom class $u$ to a differential Thom class.

Definition 3.14 Let $\hat{h}^{\bullet}$ be a multiplicative differential extension of $h^{\bullet}$. A differential Thom class of $E$ is a compactly supported class $\hat{u} \in \hat{h}_{\mathrm{cpt}}^{n}(E)$ such that $I(\hat{u}) \in h_{\mathrm{cpt}}^{n}(E)$ is a Thom class for $h^{\bullet}$.

Using the product $\hat{h}_{\mathrm{cpt}}^{\bullet}(E) \otimes_{\mathbb{Z}} \hat{h}^{\bullet}(E) \rightarrow \hat{h}_{\mathrm{cpt}}^{\bullet}(E)$, we define the differential Thom morphism, which is not surjective any more, as $\hat{\alpha} \mapsto \hat{u} \cdot \pi^{*} \hat{\alpha}$. We define the Todd class $\operatorname{Td}(\hat{u}):=\int_{E / X} R(\hat{u}) \in \Omega_{\mathrm{cl}}^{0}\left(X ; \mathfrak{h}_{\mathbb{R}}^{\bullet}\right)$. If follows that $I(\operatorname{Td}(\hat{u}))=\operatorname{Td}(I(\hat{u}))$.

Definition 3.15 Let $\pi_{X}: I \times X \rightarrow X$ be the natural projection and $i_{0}, i_{1}: X \rightarrow I \times X$ the natural embeddings. Two differential Thom classes $\hat{u}, \hat{u}^{\prime} \in \hat{h}_{\mathrm{cpt}}^{n}(E)$ are homotopic if there exists a Thom class $\hat{U} \in \hat{h}_{\mathrm{cpt}}^{n}\left(\pi_{X}^{*} E\right)$ such that $i_{0}^{*} \hat{U}=\hat{u}, i_{1}^{*} \hat{U}=\hat{u}^{\prime}$ and $\operatorname{Td}(\hat{U})=\pi_{X}^{*} \operatorname{Td}(\hat{u})$.

Let us consider two Thom classes $\hat{u}, \hat{u}^{\prime} \in \hat{h}_{\mathrm{cpt}}^{n}(E)$, refining $u \in h_{\mathrm{cpt}}^{n}(E)$. We have that $\hat{u}^{\prime}-\hat{u}=a(\eta)$, with $\eta \in \Omega_{\mathrm{cpt}}^{n-1}\left(E ; \mathfrak{h}_{\mathbb{R}}^{\bullet}\right) / \operatorname{Im} d$. Hence:

$d \int_{E / X} \eta=\int_{E / X} d \eta=\int_{E / X} R(a(\eta))=\int_{E / X}\left(R\left(\hat{u}^{\prime}\right)-R(\hat{u})\right)=\operatorname{Td}\left(\hat{u}^{\prime}\right)-\operatorname{Td}(\hat{u})$.

If $\operatorname{Td}\left(\hat{u}^{\prime}\right)=\operatorname{Td}(\hat{u})$, then $d \int_{E / X} \eta=0$ (this happens in particular if $\hat{u}$ and $\hat{u}^{\prime}$ are homotopic). In this case, there is a well-defined de-Rham cohomology class:

$$
\mathrm{dR}\left(\int_{E / X} \eta\right) \in H_{\mathrm{dR}}^{-1}\left(X ; \mathfrak{h}_{\mathbb{R}}^{\bullet}\right)
$$

Moreover, the form $\eta$ is unique up to a form $\xi$ representing a class belonging to the image of the Chern character. Because of formula (4), we have that $\mathrm{dR}(\xi) \in \operatorname{Im}(\mathrm{ch})$ if and only if $\operatorname{dR}\left(\int_{E / X} \xi\right) \in \operatorname{Td}(u) \cdot \operatorname{Im}(\mathrm{ch})$. Thus, the class (11) is uniquely determined by $\hat{u}$ and $\hat{u}^{\prime}$, up to the quotient by the subgroup $\operatorname{Td}(u) \cdot \operatorname{Im}(\mathrm{ch})$.

Definition 3.16 Let $\hat{u}$ and $\hat{u}^{\prime}$ two differential Thom classes of $\pi: E \rightarrow X$ such that $I(\hat{u})=I\left(\hat{u}^{\prime}\right)$ and $\operatorname{Td}\left(\hat{u}^{\prime}\right)=\operatorname{Td}(\hat{u})$. We define the difference class:

$$
\delta\left(\hat{u}, \hat{u}^{\prime}\right):=\left[\mathrm{dR}\left(\int_{E / X} \eta\right)\right] \in \frac{H_{\mathrm{dR}}^{-1}\left(X ; \mathfrak{h}_{\mathbb{R}}^{\bullet}\right)}{\operatorname{Td}(u) \cdot \operatorname{Imch}} .
$$

Theorem 3.17 With the hypotheses of Definition 3.16, $\hat{u}$ and $\hat{u}^{\prime}$ are homotopic if and only if $\delta\left(\hat{u}, \hat{u}^{\prime}\right)=0$. Thus, the set of homotopy classes of differential Thom classes 
on a bundle $\pi: E \rightarrow X$, refining the same topological class $u$ and with a fixed Todd class $\operatorname{Td}(\hat{u})$, is a torsor over the group:

$$
\frac{H_{\mathrm{dR}}^{-1}\left(X ; \mathfrak{h}_{\mathbb{R}}^{\bullet}\right)}{\operatorname{Imch} \cdot \operatorname{Td}(u)} .
$$

For the proof see [3, pp. 125-126]. The following corollary extends Lemma 3.1 to the differential case.

Corollary $3.18(2 \times 3$ principle) Given two bundles $E, F \rightarrow X$, with projections $p_{E}: E \oplus F \rightarrow E$ and $p_{F}: E \oplus F \rightarrow F$, we consider a triple $(\hat{u}, \hat{v}, \hat{w})$ of differential Thom classes on $E, F$ and $E \oplus F$ respectively, such that $\hat{w}$ is homotopic to $p_{E}^{*} \hat{u} \cdot p_{F}^{*} \hat{v}$. Two elements of such a triple uniquely determine the third one up to homotopy.

Proof Let us suppose that $\hat{w}$ and $\hat{u}$ are fixed up to homotopy. We choose a topological Thom class $v$ on $F$ such that $p_{E}^{*} u \oplus p_{E}^{*} v=w$. Let $\hat{v}^{\prime}$ be any differential refinement of $v$ with Todd class $\operatorname{Td}(\hat{v})=\operatorname{Td}(\hat{u})^{-1} \cdot \operatorname{Td}(\hat{w})$. The class $\hat{v}$ we are looking for must be of the form $\hat{v}:=\hat{v}^{\prime}-a(\eta)$, with $d \int_{E / X} \eta=0$. The homotopy class of $\hat{v}$ is completely determined by $\left[\mathrm{dR}\left(\int_{E / X} \eta\right)\right]$. We have that $\delta(\hat{u} \hat{v}, \hat{w})=\delta\left(\hat{u} \hat{v}^{\prime}, \hat{w}\right)-$ $\left[\mathrm{dR}\left(\int_{E \oplus F / X} p_{F}^{*} R(\hat{u}) \cdot p_{E}^{*} \eta\right)\right]=\delta\left(\hat{u} \hat{v}^{\prime}, \hat{w}\right)-[\operatorname{Td}(u)]\left[\mathrm{dR}\left(\int_{E / X} \eta\right)\right]$, hence the only possibility is $\left[\mathrm{dR}\left(\int_{E / X} \eta\right)\right]=[\operatorname{Td}(u)]^{-1} \cdot \delta\left(\hat{u} \hat{v}^{\prime}, \hat{w}\right)$.

Finally, we will need the following corollary of Theorem 3.17.

Corollary 3.19 On the trivial bundle $X \times \mathbb{R}^{N}$ there is a canonical homotopy class of differential Thom classes, refining the canonical topological one.

Proof Let us consider the line bundle $\{p t\} \times \mathbb{R}$. We put the canonical Thom class $u_{0}$, defined as the suspension of $1 \in \mathfrak{h}^{0}$. For any refinement, the Todd class is necessary 1 . We choose $\hat{u}_{0}$ in such a way that $\int_{S^{1}} \hat{u}_{0}=1$, identifying $S^{1}$ with $\mathbb{R}^{+}$. We show that $\hat{u}_{0}$ exists and is unique up to homotopy. Let $\hat{u}_{0}^{\prime}$ be any differential refinement of $u_{0}$. Then $\int_{S^{1}} \hat{u}_{0}^{\prime}=1+a(x)$, therefore we choose $\hat{u}_{0}:=\hat{u}_{0}^{\prime}-a(x d t)$. If $\hat{u}_{0}$ and $\hat{u}_{0}^{\prime}$ both satisfy $\int_{S^{1}} \hat{u}_{0}=\int_{S^{1}} \hat{u}_{0}^{\prime}$, then $\hat{u}_{0}^{\prime}=\hat{u}_{0}+a(\eta)$ with $a\left(\int_{S^{1}} \eta\right)=0$, i.e., $\int_{S^{1}} \eta \in \operatorname{Im}(\mathrm{ch})$. Hence, $\delta\left(\hat{u}_{0}, \hat{u}_{0}^{\prime}\right)=0$, thus $\hat{u}_{0}$ is unique up to homotopy. On the bundle $\{p t\} \times \mathbb{R}^{N}$ we put the class $\hat{u}_{0, N}=\pi_{1}^{*} \hat{u}_{0} \cdots \pi_{N}^{*} \hat{u}_{0}$ and, on the bundle $X \times \mathbb{R}^{N}$, the class $\pi_{\mathbb{R}^{N}}^{*} \hat{u}_{0, N}$.

\subsection{Differential integration}

The Gysin map $f_{!}: \hat{h}^{\bullet}(Y) \rightarrow \hat{h}^{\bullet-n}(X)$, for $n=\operatorname{dim} Y-\operatorname{dim} X$, is defined similarly to (5), starting from the same data and refining the Thom class of the normal bundle to a differential one:

$$
f_{!}(\hat{\alpha})=\int_{\mathbb{R}^{N}} i_{*} \varphi_{*}\left(\hat{u} \cdot \pi^{*} \hat{\alpha}\right) .
$$


The integration map $\int_{\mathbb{R}^{N}}: \hat{h}_{\mathrm{cpt}}^{\bullet+N}\left(X \times \mathbb{R}^{N}\right) \rightarrow \hat{h}^{\bullet}(X)$ is defined as follows. There is a natural projection $\pi^{N}:\left(S^{1}\right)^{N} \rightarrow S^{N}$, defined thinking of $S^{N}$ as $S^{1} \wedge \ldots \wedge S^{1}=$ $\left(S^{1}\right)^{N} /\left(S^{1} \vee \ldots \vee S^{1}\right)$. For $\hat{\alpha} \in \hat{h}^{n+N}\left(X \times S^{N}\right)$, we define:

$$
\int_{S^{n}} \hat{\alpha}:=\int_{S^{1}} \cdots \int_{S^{1}}\left(\mathrm{id} \times \pi^{N}\right)^{*} \hat{\alpha} .
$$

Given a class $\hat{\alpha} \in \hat{h}_{\mathrm{cpt}}^{n+N}\left(X \times \mathbb{R}^{N}\right)$, since $S^{N}$ is the one-point compactification of $\mathbb{R}^{N}$, considering the embedding $j: \mathbb{R}^{N} \hookrightarrow S^{N}$ we can naturally define $j_{*} \hat{\alpha} \in$ $\hat{h}^{n+N}\left(X \times S^{N}\right)$ and $\int_{\mathbb{R}^{N}} \hat{\alpha}:=\int_{S^{N}} j_{*} \hat{\alpha}$. We remark that, for any $N_{1}, N_{2}$ such that $N_{1}+N_{2}=N$, we have $\int_{\mathbb{R}^{N}} \hat{\alpha}=\int_{\mathbb{R}^{N_{2}}} \int_{\mathbb{R}^{N_{1}}} \hat{\alpha}$. That's because, $\hat{\alpha}$ being compactly supported, its extensions commute with any map between compactifications of $\mathbb{R}^{N}$; since $\left(S^{1}\right)^{N} \simeq\left(S^{1}\right)^{N_{2}} \times\left(S^{1}\right)^{N_{1}}$, we get the result.

We define a representative of an $\hat{h}^{\bullet}$-orientation of $f$ as in Definition 3.2 , but considering a differential Thom class. Fixing such a representative $(\iota, \hat{u}, \varphi)$, the Gysin map $f_{\text {! }}$ is well-defined. Moreover, there is a natural map on differential forms, called curvature map:

$$
\begin{aligned}
& R_{(\iota, \hat{u}, \varphi)}: \Omega^{\bullet}\left(Y ; \mathfrak{h}_{\mathbb{R}}^{\bullet}\right) \rightarrow \Omega^{\bullet-n}\left(X ; \mathfrak{h}_{\mathbb{R}}^{\bullet}\right) \\
& \omega \mapsto \int_{X \times \mathbb{R}^{N} / X} i_{*} \varphi_{*}\left(R(\hat{u}) \wedge \pi^{*} \omega\right) .
\end{aligned}
$$

It is easy to prove from the axioms that:

$$
R(f ! \hat{\alpha})=R_{(\iota, \hat{u}, \varphi)}(R(\hat{\alpha})) \quad f ! a(\omega)=a\left(R_{(\iota, \hat{u}, \varphi)}(\omega)\right) .
$$

The following definition is analogous to Definition 3.5, but it takes into account the curvature map. We define proper representatives as in the topological framework.

Definition 3.20 A homotopy between two representatives $(\iota, \hat{u}, \varphi)$ and $\left(\iota^{\prime}, \hat{u}^{\prime}, \varphi^{\prime}\right)$ of an $\hat{h}^{\bullet}$-orientation of $f: Y \rightarrow X$ is a representative $(J, \hat{U}, \Phi)$ of an $\hat{h}^{\bullet}$-orientation of id $\times f: I \times Y \rightarrow I \times X$, such that:

- $(J, I(\hat{U}), \Phi)$ is proper over a neighborhood $V \subset I$ of $\{0,1\}$;

- $\left.(J, \hat{U}, \Phi)\right|_{f_{0}}=(\iota, \hat{u}, \varphi)$ e $\left.(J, \hat{U}, \Phi)\right|_{f_{1}}=\left(\iota^{\prime}, \hat{u}^{\prime}, \varphi^{\prime}\right)$;

- $\pi_{X}^{*} \circ R_{(\iota, \hat{u}, \varphi)}=R_{(J, \hat{U}, \Phi)} \circ \pi_{Y}^{*}$.

In particular, it follows that $R_{(\iota, \hat{u}, \varphi)}=R_{\left(\iota^{\prime}, \hat{u}^{\prime}, \varphi^{\prime}\right)}$. The following lemma is easy to prove.

Lemma 3.21 Let $(\iota, \hat{u}, \varphi) e\left(\iota, \hat{u}^{\prime}, \varphi\right)$ be two proper representatives such that $\hat{u}$ and $\hat{u}^{\prime}$ are homotopic as differential Thom classes. Then the two representatives are homotopic.

Thanks to Corollary 3.19, we define the equivalence of representatives up to stabilization as in the topological framework (Definition 3.6). 
Definition 3.22 An $\hat{h}^{\bullet}$-orientation on $f: Y \rightarrow X$ is an equivalence class $[\iota, \hat{u}, \varphi]$ of representatives, up to the equivalence relation generated by homotopy and stabilization.

As a consequence of formula (2), $f$ ! only depends on the $\hat{h}^{\bullet}$-orientation of $f$, not on the specific representative [3, sec. 4.10]. We now consider a submersion $f: Y \rightarrow X$. In this case the Gysin map provides a good notion of integration.

Lemma 3.23 Let $f: Y \rightarrow X$ be a submersion. Then, for any neat embedding $\iota: Y \hookrightarrow X \times \mathbb{R}^{N}$ and any Thom class $\hat{u}$ of the normal bundle, there exists a proper representative $(\iota, \hat{u}, \varphi)$ of an $\hat{h}^{\bullet}$-orientation of $f$. Moreover, any two such representatives are homotopic, hence we can denote the orientation by $[\iota, \hat{u}]$.

Proof The proof of the first statement is the same as Theorem 3.4. Because of the uniqueness up to homotopy of the tubular neighborhood and the embedding, we can find a homotopy (id $\left.\times \iota, \pi_{I}^{*} \hat{u}, \Phi\right)$ between the two representatives, which is proper. We have:

$$
\begin{aligned}
R_{\left(\mathrm{id} \times \iota, \pi_{I}^{*} \hat{u}, \Phi\right)}\left(\pi_{Y}^{*} \omega\right) & =\int_{I \times X \times \mathbb{R}^{N} / I \times X} I_{*} \Phi_{*}\left(\pi_{I}^{*} R(\hat{u}) \cdot \pi^{*} \omega\right) \\
& =\pi_{X}^{*} \int_{N_{\iota(Y)}\left(X \times \mathbb{R}^{N}\right)} R(\hat{u}) \cdot \pi^{*} \omega=\pi_{X}^{*}\left(R_{(\iota, \hat{u}, \varphi)}(\omega)\right) .
\end{aligned}
$$

Lemma 3.24 If the representative $(\iota, \hat{u}, \varphi)$ is proper, then the Gysin map is a morphism of $\hat{h}^{\bullet}(X)$-modules, i.e., for every $\hat{\alpha} \in \hat{h}^{\bullet}(Y)$ and $\hat{\beta} \in \hat{h}^{\bullet}(X)$ :

$$
f_{!}\left(\hat{\alpha} \cdot f^{*}(\hat{\beta})\right)=f_{!}(\hat{\alpha}) \cdot \hat{\beta}
$$

Proof

$$
\begin{aligned}
f_{!}\left(\hat{\alpha} \cdot f^{*} \hat{\beta}\right) & =\int_{\mathbb{R}^{N}} l_{!}\left(\hat{\alpha} \cdot f^{*} \hat{\beta}\right)=\int_{\mathbb{R}^{N}} i_{*} \varphi_{*}\left(\hat{u} \cdot \pi^{*} \hat{\alpha} \cdot \pi^{*} \iota^{*} \pi_{X}^{*} \hat{\beta}\right) \\
& =\int_{\mathbb{R}^{N}} i_{*} \varphi_{*}\left(\hat{u} \cdot \pi^{*} \hat{\alpha} \cdot \pi^{*}\left(\left.\pi_{X}\right|_{\iota(Y)}\right)^{*} \hat{\beta}\right) \\
& =\int_{\mathbb{R}^{N}} i_{*}\left(\varphi_{*}\left(\hat{u} \cdot \pi^{*} \hat{\alpha}\right) \cdot\left(\varphi_{*} \pi^{*}\left(\left.\pi_{X}\right|_{\iota(Y)}\right)^{*} \hat{\beta}\right)\right) \\
& \stackrel{(6)}{=} \int_{\mathbb{R}^{N}} i_{*}\left(\varphi_{*}\left(\hat{u} \cdot \pi^{*} \hat{\alpha}\right) \cdot\left(\left.\pi_{X}\right|_{U} ^{*} \hat{\beta}\right)\right) \\
& =\int_{\mathbb{R}^{N}} i_{*}\left(\varphi_{*}\left(\hat{u} \cdot \pi^{*} \hat{\alpha}\right)\right) \cdot \pi_{X}^{*} \hat{\beta}=\left(\int_{\mathbb{R}^{N}} \iota(\hat{\alpha})\right) \cdot \hat{\beta}=f_{!}(\hat{\alpha}) \cdot \hat{\beta} .
\end{aligned}
$$


Lemma 3.25 If $(\iota, \hat{u}, \varphi)$ is proper, then:

$$
R_{(\iota, \hat{u}, \varphi)}(\omega)=\int_{Y / X} \operatorname{Td}(\hat{u}) \wedge \omega .
$$

Proof Since the representative is proper, the tubular neighborhood $U$ is fibered over $\iota(Y)$, with fibers contained in $\mathbb{R}^{N}$; moreover, the disjoint union of the fibers over $\iota\left(f^{-1}\{x\}\right)$ is a fiber over $x$. Hence, integrating with respect to $X \times \mathbb{R}^{N} / X$ a form, whose support is contained in $U$, is equivalent to integrating over $U / \iota(Y)$ and then over $Y / X$. Hence, we have:

$$
\begin{aligned}
R_{(\iota, \hat{u}, \varphi)} \omega & =\int_{X \times \mathbb{R}^{N} / X} i_{*} \varphi_{*}\left(R(\hat{u}) \wedge \pi^{*} \omega\right)=\int_{Y / X} \int_{U / \iota(Y)} \varphi_{*}\left(R(\hat{u}) \wedge \pi^{*} \omega\right) \\
& =\int_{Y / X}\left(\int_{N_{\iota(Y)} / \iota(Y)} R(\hat{u})\right) \wedge \omega=\int_{Y / X} \operatorname{Td}(\hat{u}) \wedge \omega .
\end{aligned}
$$

Corollary 3.26 If $(\iota, \hat{u}, \varphi)$ is proper, then:

$$
R\left(f_{!} \hat{\alpha}\right)=\int_{Y / X} \operatorname{Td}(\hat{u}) \wedge R(\hat{\alpha}) \quad f_{!} a(\omega)=a\left(\int_{Y / X} \operatorname{Td}(\hat{u}) \wedge \omega\right)
$$

Proof This immediately follows from lemma 3.25.

Lemma 3.27 Let $f: Y \rightarrow X$ and $g: X \rightarrow W$ be $h^{\bullet}$-oriented neat submersions. There is a naturally induced $h^{\bullet}$-orientation on $g \circ f: Y \rightarrow W$ such that $(g \circ f) !=$ $g$ ! $\circ$ ! .

Proof We call $[\iota, u]$ and $[\kappa, v]$ the orientations of $f$ and $g$, and we construct an orientation $[\xi, w]$ on $g \circ f$ as in Definition 3.9. The reader can prove that it does not depend on the representatives chosen for $[\iota, u]$ and $[\kappa, v]$. It remains to show that $(g \circ f) !=g_{!} \circ f_{!}$. In order to prove this, we choose tubular neighborhoods $U$ and $V$ and diffeomorphisms $\varphi: N_{\iota(Y)}\left(X \times \mathbb{R}^{N}\right) \rightarrow U$ and $\psi: N_{\kappa(X)}\left(W \times \mathbb{R}^{L}\right) \rightarrow V$, in such a way that the representatives $(\iota, u, \varphi)$ and $(\kappa, v, \psi)$ are proper. We define the proper representative $(\xi, w, v)$ in the following way:

- in order to define the tubular neighborhood of $\xi(Y)$ in $W \times \mathbb{R}^{L+N}$, for each point of the image of $\varphi$ in $X \times \mathbb{R}^{N}$, we consider the image under $\left(\psi, \mathrm{id}_{\mathbb{R}^{N}}\right)$ of the corresponding fiber of $N_{\kappa(X) \times \mathbb{R}^{N}}\left(W \times \mathbb{R}^{L+N}\right) \simeq \pi_{N}^{*} N_{\kappa(X)}\left(W \times \mathbb{R}^{L}\right) \simeq$ $N_{\kappa(X)}\left(W \times \mathbb{R}^{L}\right) \times \mathbb{R}^{N}$;

- $v$ is defined in the following way: given a vector $(A, B)_{y} \in N_{\iota(Y)}\left(X \times \mathbb{R}^{N}\right) \oplus$ $\left.\left(\pi_{N}^{*} N_{\kappa(X)}\left(W \times \mathbb{R}^{L}\right)\right)\right|_{\xi(Y)}$, we apply $\varphi$ to $A$ in $y$ getting a point $p \in\{f(y)\} \times \mathbb{R}^{N}$; since $\pi_{N}^{*} N_{\kappa(X)}\left(W \times \mathbb{R}^{L}\right)$, restricted to $\{f(y)\} \times \mathbb{R}^{N}$, coincides with $\left(N_{\kappa(X)}(W \times\right.$ $\left.\left.\mathbb{R}^{L}\right)\right)_{\kappa(f(y))} \times \mathbb{R}^{N}$, we apply $\left(\psi, 1_{\mathbb{R}^{N}}\right)$ to $B$, the latter translated from $\xi(y)$ to $(f(y), p)$, and we get $v(A, B)$. 
Basically the parallel translation along $\mathbb{R}^{N}$ allows us to identify the bundle $\left.\left(N_{\kappa(X) \times \mathbb{R}^{N}}\left(W \times \mathbb{R}^{L+N}\right)\right)\right|_{\kappa(U)}, U$ being the image of $\varphi$, with the "propagation" along $U$ of $\left.\left(N_{\kappa(X) \times \mathbb{R}^{N}}\left(W \times \mathbb{R}^{N+L}\right)\right)\right|_{\xi(Y)}$. By definition, the orientation of $\left(N_{\kappa(X) \times \mathbb{R}^{N}}(W \times\right.$ $\left.\left.\mathbb{R}^{L+N}\right)\right)\left.\right|_{\kappa(U)}$ coincides with the "propagation" of the orientation of $\left(N_{\kappa(X) \times \mathbb{R}^{N}}(W \times\right.$ $\left.\left.\mathbb{R}^{N+L}\right)\right)\left.\right|_{\xi(Y)}$. Let us consider the following diagram:

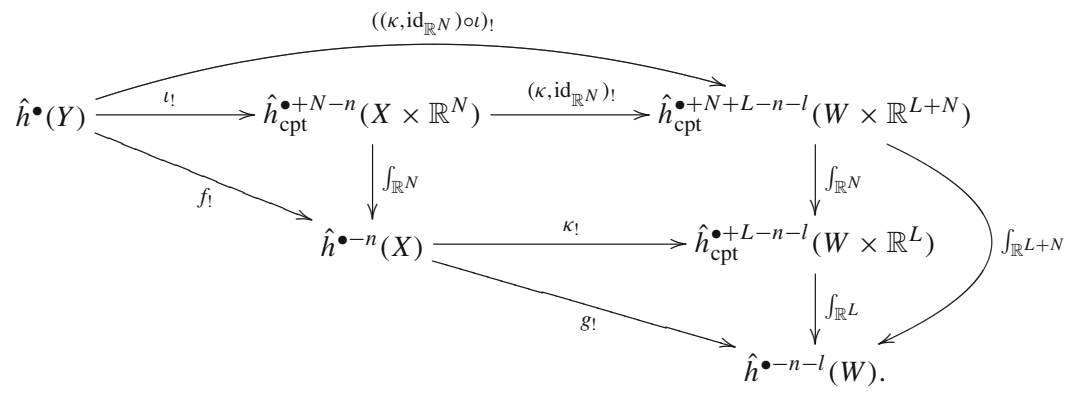

The curved arrows define $(g \circ f)$ !, therefore we have to prove the commutativity of the diagram. The left and lower triangles commute by definition. Commutativity of the right cell is a property of integration that we already remarked. About the upper cell, one has: ${ }^{2}$

$$
\begin{aligned}
\left(\left(\kappa, \mathrm{id}_{\mathbb{R}^{N}}\right) \circ \iota\right) ! \hat{\alpha} & =\psi_{v}^{*} \nu_{*}\left(\hat{\alpha} \cdot p_{1}^{*} \hat{u} \cdot p_{2}^{*}\left(\left.\left(\pi_{N}^{*} \hat{v}\right)\right|_{\xi(Y)}\right)\right) \\
& \left.=\psi_{\pi_{N}^{*} \psi}^{*}\left(\pi_{N}^{*} \psi\right)_{*}\left(\left.\psi_{\varphi}^{*} \varphi_{*}\left(\pi^{*} \hat{\alpha} \cdot \hat{u}\right) \cdot\left(\pi_{N}^{*} \hat{v}\right)\right|_{\xi(Y)}\right)\right) \\
& =\psi_{\pi_{N}^{*} \psi}^{*}\left(\pi_{N}^{*} \psi\right)_{*}\left(\left.\pi^{*} \iota ! \alpha \cdot\left(\pi_{N}^{*} \hat{v}\right)\right|_{V}\right) \\
& =\left(\kappa, \mathrm{id}_{\mathbb{R}^{N}}\right) ! \iota ! \alpha .
\end{aligned}
$$

Finally, about the central square:

$$
\begin{aligned}
\int_{\mathbb{R}^{N}}\left(\kappa, \mathrm{id}_{\mathbb{R}^{N}}\right) \hat{\alpha} & =\int_{\mathbb{R}^{N}}\left(\pi_{N}^{*} j\right)_{*}\left(\pi_{N}^{*} \psi\right)_{*}\left(\pi^{*} \hat{\alpha} \cdot \pi_{N}^{*} \hat{v}\right)=j_{*} \psi_{*}\left(\int_{\mathbb{R}^{N}} \pi^{*} \hat{\alpha} \cdot \pi_{N}^{*} \hat{v}\right) \\
& =j_{*} \psi_{*}\left(\left(\int_{\mathbb{R}^{N}} \pi^{*} \hat{\alpha}\right) \cdot \pi_{N}^{*} \hat{v}\right)=\kappa !\left(\int_{\mathbb{R}^{N}} \hat{\alpha}\right) .
\end{aligned}
$$

The following lemma is a consequence of Lemma 3.18 and of the uniqueness up to homotopy and stabilization of the embedding $\iota$.

Lemma $3.28\left(2 \times 3\right.$ principle) Let $f: Y \rightarrow X$ and $g: X \rightarrow W$ be $\hat{h}^{\bullet}$-oriented neat submersions, with orientations $[\iota, \hat{u}]$ and $[\kappa, \hat{v}]$, and let $[\xi, \hat{w}]$ be the orientation induced on $g \circ f$, as shown in Lemma 3.27. Two elements of the triple $([\iota, \hat{u}],[\kappa, \hat{v}],[\xi, \hat{w}])$ uniquely determines the third one.

\footnotetext{
${ }^{2}$ In the third line of the following equation we should have $\pi_{N}^{*} \hat{v}$, without the restriction to $V$, since $V$ does not appear in the definition of $\left(\kappa, \mathrm{id}_{\mathbb{R}^{N}}\right)$ ! . Hence, we are actually calculating $\left(\left.\left(\kappa, \operatorname{id}_{\mathbb{R}^{N}}\right)\right|_{V}\right)$ ! . Nevertheless, since $\iota \grave{\alpha}$ vanishes outside $V$, the restriction is immaterial.
} 
Finally, we consider differential orientations of manifolds. We define them as in the topological case (Definitions 3.12, 3.13). We put $\operatorname{Td}(X):=\operatorname{Td}(\hat{u}), \hat{u}$ being the orientation of the stable normal bundle. If follows from Corollary 3.26 that:

$$
R\left(\left(p_{X}\right) !(\hat{\alpha})\right)=\int_{X} \operatorname{Td}(X) \wedge R(\hat{\alpha}) .
$$

Let us consider a submersion $f: Y \rightarrow X$ between compact $\hat{h}^{\bullet}$-oriented manifolds. Since $p_{Y}=p_{X} \circ f$, it follows from Lemma 3.28 that $f$ inherits a unique orientation from the ones of $X$ and $Y$. Hence, the integration map $f_{!}: \hat{h}^{\bullet}(Y) \rightarrow \hat{h}^{\bullet-n}(X)$, for $n=\operatorname{dim} Y-\operatorname{dim} X$, is well-defined for submersions between compact $\hat{h}^{\bullet}$-oriented manifolds.

Lemma 3.29 For $X$ an $\hat{h}^{\bullet}$-manifold with boundary and $\Phi: X \rightarrow I$ defined as in Definition 3.13, we have:

$$
\int_{0}^{1} R(\Phi, \hat{\alpha})=\int_{X} \operatorname{Td}(X) \wedge R(\hat{\alpha}) .
$$

Proof Because of formula (14), we have:

$$
\begin{aligned}
\int_{0}^{1} R(\Phi, \hat{\alpha}) & =\int_{0}^{1} \int_{I \times \mathbb{R}^{N} / I} i_{*} \varphi_{*}\left(R(\hat{u}) \wedge \pi^{*} R(\hat{\alpha})\right)=\int_{N_{\iota(X)}\left(I \times \mathbb{R}^{N}\right)} R(\hat{u}) \wedge \pi^{*} R(\hat{\alpha}) \\
& =\int_{X}\left(\int_{N_{l(X)}\left(I \times \mathbb{R}^{N}\right) / \iota(X)} R(\hat{u})\right) \wedge R(\hat{\alpha})=\int_{X} \operatorname{Td}(X) \wedge R(\hat{\alpha}) .
\end{aligned}
$$

About the restriction of an orientation to the boundary, the same considerations we did in the topological framework hold for differential extensions. In particular, formula (9) keeps on holding.

\section{Cohomology and homology}

Let us consider a multiplicative cohomology theory $h^{\bullet}$ represented by a spectrum $\left(E_{\bullet}, e_{\bullet}, \varepsilon_{\bullet}\right)$, where $e_{n}$ is the marked point of $E_{n}$ and $\varepsilon_{n}:\left(\Sigma E_{n}, \Sigma e_{n}\right) \rightarrow\left(E_{n+1}, e_{n+1}\right)$ is the structure map, whose adjoint $\tilde{\varepsilon}_{n}: E_{n} \rightarrow \Omega_{e_{n+1}} E_{n+1}$ is a homotopy equivalence. Considering the spectrum $\left(E_{\bullet} \wedge X, e_{\bullet} \wedge x_{0}, \varepsilon_{\bullet} \wedge 1\right)$, the dual homology theory $h_{\bullet}$ is defined, on a space with marked point $\left(X, x_{0}\right)$, as [17]:

$$
h_{n}\left(X, x_{0}\right):=\pi_{n}\left(E_{\bullet} \wedge X, e_{\bullet} \wedge x_{0}\right)=\underset{k}{\lim } \pi_{n+k}\left(E_{k} \wedge X, e_{k} \wedge x_{0}\right) .
$$

The unreduced groups are defined as $h_{n}(X):=h_{n}\left(X_{+}, \infty\right)$, for $X_{+}=X \sqcup\{\infty\}$. There is a natural map for every $n \in \mathbb{Z}$ [15, pp. 289-290]:

$$
\xi^{n}: h^{n}(X) \rightarrow \operatorname{Hom}_{\mathfrak{h}} \bullet\left(h_{n-\bullet}(X), \mathfrak{h}^{\bullet}\right)
$$


From (17) we can easily define:

$$
\xi_{\mathbb{R}}^{n}: h^{n}(X) \otimes_{\mathbb{Z}} \mathbb{R} \rightarrow \operatorname{Hom}_{\mathfrak{h}}\left(h_{n-\bullet}(X), \mathfrak{h}_{\mathbb{R}}^{\bullet}\right)
$$

It follows from the universal coefficient theorem (see [1, prop. 13.5, p. 285] and $\left[15\right.$, p. 290]) that the map (18) is an isomorphism. Finally, for $\mathfrak{h}_{\mathbb{R} / \mathbb{Z}}^{\bullet}:=h^{\bullet}(\{*\} ; \mathbb{R} / \mathbb{Z})$, there is a natural map:

$$
\xi_{\mathbb{R} / \mathbb{Z}}^{n}: h^{n}(X ; \mathbb{R} / \mathbb{Z}) \rightarrow \operatorname{Hom}_{\mathfrak{h}} \bullet\left(h_{n-\bullet}(X), \mathfrak{h}_{\mathbb{R} / \mathbb{Z}}^{\bullet}\right)
$$

For singular cohomology or K-theory (19) is an isomorphism, as a consequence of the universal coefficient theorem $[13,18]$. This depends on the fact that ordinary cohomology and K-theory are Pontrjagin self-dual [6].

In [9] the author provides a geometric construction of the homology theory dual to a given cohomology theory, which we briefly recall in the following, only in the case of a single space $X$. With respect to [9], in the following definition we replace the quotient by diffeomorphisms and vector bundle modification with the quotient by the Gysin map associated to a submersion. Actually, we could not require that the map is a submersion, but this hypothesis will make it easier to define the differential extension of cycles.

Definition 4.1 Let $h^{\bullet}$ be a multiplicative cohomology theory. On a space $X$ with the homotopy type of a finite CW-complex, we define:

- the group of $n$-precycles of $h_{\bullet}$ as the free abelian group generated by the quadruples $(M, u, \alpha, f)$, with:

- $(M, u)$ a smooth compact manifold (without boundary) with $h^{\bullet}$-orientation $u$, whose connected components $\left\{M_{i}\right\}$ have dimension $n+q_{i}$, with $q_{i}$ arbitrary;

- $\alpha \in h^{\bullet}(M)$, such that $\left.\alpha\right|_{M_{i}} \in h^{q_{i}}(M)$;

- $f: M \rightarrow X$ a continuous map;

- the group of $n$-cycles of $h_{\bullet}$, denoted by $z_{n}(X)$, as the quotient of the group of $n$-precycles by the free subgroup generated by elements of the form:

- $(M, u, \alpha+\beta, f)-(M, u, \alpha, f)-(M, u, \beta, f)$;

- $(M, u, \alpha, f)-\left(M_{1},\left.u\right|_{M_{1}},\left.\alpha\right|_{M_{1}},\left.f\right|_{M_{1}}\right)-\left(M_{2},\left.u\right|_{M_{2}},\left.\alpha\right|_{M_{2}},\left.f\right|_{M_{2}}\right)$, for $M=$ $M_{1} \sqcup M_{2} ;$

- $(M, u, \varphi ! \alpha, f)-(N, v, \alpha, f \circ \varphi)$ for $\varphi: N \rightarrow M$ a submersion and $\varphi$ ! : $h^{\bullet}(N) \rightarrow h^{\bullet}(M)$ the Gysin map;

- the group of $n$-boundaries of $h_{\bullet}$, denoted by $b_{n}(X)$, as the subgroup of $z_{n}(X)$ generated by the cycles which are representable by a pre-cycle $(M, u, \alpha, f)$, such that there exits a quadruple ( $W, U, A, F)$, where $W$ is a manifold and $M=\partial W, U$ is an $h^{\bullet}$-orientation of $W$ and $\left.U\right|_{M}=u, A \in h^{\bullet}(W)$ and $\left.A\right|_{M}=\alpha, F: W \rightarrow X$ is a continuous map satisfying $\left.F\right|_{M}=f$.

We define $h_{n}(X):=z_{n}(X) / b_{n}(X)$.

Describing in this way the dual homology theory, the map (17) corresponds to:

$$
\begin{gathered}
\xi^{n}: h^{n}(X) \rightarrow \operatorname{Hom}_{\mathfrak{h}}\left(h_{n-\bullet}(X), \mathfrak{h}^{\bullet}\right) \\
\alpha \mapsto\left([M, u, \beta, f] \mapsto\left(p_{M}\right) !\left(\beta \cdot f^{*} \alpha\right)\right),
\end{gathered}
$$


where $p_{M}: M \rightarrow\{p t\}$. We verify that (20) is well-defined. If we consider a function between compact manifolds $\varphi: N \rightarrow M$ and two representatives $\left(M, u, \varphi_{!} \beta, f\right)$ and $(N, v, \beta, f \circ \varphi)$ of the homology class, we have:

$$
\begin{aligned}
\xi^{n}(\alpha)[N, v, \beta, f \circ \varphi] & =\left(p_{N}\right)_{!}\left(\beta \cdot \varphi^{*} f^{*} \alpha\right)=\left(p_{M}\right) ! \varphi !\left(\beta \cdot \varphi^{*} f^{*} \alpha\right) \\
& =\left(p_{M}\right) !\left(\varphi ! \beta \cdot f^{*} \alpha\right)=\xi^{n}(\alpha)[M, u, \varphi ! \beta, f]
\end{aligned}
$$

Let us now suppose that $(M, u, \beta, f)=\partial(W, U, B, F)$. Then we consider a function $\Phi: W \rightarrow[0,1]$, such that $\Phi^{-1}(0)=M$ and $\Phi^{-1}(1)=\emptyset$. We have that $\left(p_{M}\right)_{!}\left(\beta \cdot f^{*} \alpha\right)=\left.\Phi_{!}\left(B \cdot F^{*} \alpha\right)\right|_{\{0\}}$. By homotopy invariance, such a class coincides with $\left.\Phi_{!}\left(B \cdot F^{*} \alpha\right)\right|_{\{1\}}=0$. Finally, the image of $\alpha$ is a $\mathfrak{h}^{\bullet}$-module homomorphism, since, for $\gamma \in \mathfrak{h}^{t}$ :

$$
\begin{aligned}
\xi^{n}(\alpha)([(M, u, \beta, f)] \cdot \gamma) & =\xi^{n}(\alpha)\left[M, u, \beta \cdot\left(p_{M}\right)^{*} \gamma, f\right]=\left(p_{M}\right) !\left(\beta \cdot f^{*} \alpha \cdot\left(p_{M}\right)^{*} \gamma\right) \\
& =\left(p_{M}\right) !\left(\beta \cdot f^{*} \alpha\right) \cdot \gamma=\xi^{n}(\alpha)[M, u, \beta, f] \cdot \gamma
\end{aligned}
$$

Tensorizing with $\mathbb{R}$, we get the isomorphism:

$$
\xi_{\mathbb{R}}^{n}: h^{n}(X) \otimes_{\mathbb{Z}} \mathbb{R} \stackrel{\simeq}{\longrightarrow} \operatorname{Hom}_{\mathfrak{h}} \cdot\left(h_{n-\bullet}(X), \mathfrak{h}_{\mathbb{R}}^{\bullet}\right) .
$$

The map (19) corresponds to:

$$
\begin{array}{r}
\xi_{\mathbb{R} / \mathbb{Z}}^{n}: h^{n}(X ; \mathbb{R} / \mathbb{Z}) \rightarrow \operatorname{Hom}_{\mathfrak{h}}\left(h_{n-\bullet}(X), \mathfrak{h}_{\mathbb{R} / \mathbb{Z}}^{\bullet}\right) \\
\alpha \mapsto\left([M, u, \beta, f] \mapsto\left(p_{M}\right)_{!}\left(\beta \cdot f^{*} \alpha\right)\right) .
\end{array}
$$

The product $\beta \cdot f^{*} \alpha$ is provided by the structure of $h^{\bullet}$-module on $h^{\bullet}(\cdot ; \mathbb{R} / \mathbb{Z})$.

\section{Flat pairing}

\subsection{Flat classes}

Given a smooth map $f: Y \rightarrow X$, with $n=\operatorname{dim} Y-\operatorname{dim} X$, the Gysin map $f !$ : $\hat{h}^{\bullet}(Y) \rightarrow \hat{h}^{\bullet-n}(X)$ previously defined depends on the $\hat{h}^{\bullet}$-orientation, but, if we restrict to flat classes, it only depends on the topological $h^{\bullet}$-orientation. In order to prove this statement, we show that there is a natural graded module structure on $\hat{h}_{\mathrm{fl}}^{\bullet}(X)$ over $h^{\bullet}(X)$, i.e. there exists a product:

$$
h^{\bullet}(X) \otimes_{\mathbb{Z}} \hat{h}_{\mathrm{fl}}^{\bullet}(X) \rightarrow \hat{h}_{\mathrm{fl}}^{\bullet}(X) .
$$

This is due to the fact that the product of differential classes restricts to a product $\hat{h}^{\bullet}(X) \otimes_{\mathbb{Z}} \hat{h}_{\mathrm{fl}}^{\bullet}(X) \rightarrow \hat{h}_{\mathrm{fl}}^{\bullet}(X)$, since, the curvature being multiplicative, if one of the two factors has vanishing curvature, also the result has. Moreover, the product $\hat{\alpha} \cdot \hat{\beta}$, with $\hat{\beta}$ flat, only depends on $I(\hat{\alpha})$. In fact, if $I(\hat{\alpha})=0$, then $\hat{\alpha}=a(\omega)$. Because of Definition 2.4, we have $a(\omega) \cdot \hat{\beta}=a(\omega \wedge R(\hat{\beta}))=0$. We can show in the same way that 
also the product $\hat{h}_{\mathrm{cpt}}^{\bullet}(E) \otimes_{\mathbb{Z}} \hat{h}^{\bullet}(E) \rightarrow \hat{h}_{\mathrm{cpt}}^{\bullet}(E)$ can be refined to $h_{\mathrm{cpt}}^{\bullet}(E) \otimes_{\mathbb{Z}} \hat{h}_{\mathrm{fl}}^{\bullet}(E) \rightarrow$ $\hat{h}_{\mathrm{fl}, \mathrm{cpt}}^{\bullet}(E)$. Therefore, given a real vector bundle $E \rightarrow X$ of rank $n$ with (topological) Thom class $u$, we define the Thom morphism:

$$
\begin{aligned}
T_{\mathrm{fl}}: \hat{h}_{\mathrm{fl}}^{\bullet}(X) & \rightarrow \hat{h}_{\mathrm{fl}, \mathrm{cpt}}^{\bullet+n}(E) \\
\hat{\alpha} & \mapsto u \cdot \pi^{*} \hat{\alpha} .
\end{aligned}
$$

From this it easily follows that the Gysin map $f_{!}$, when applied to a flat class, only depends on the topological orientation. Lemmas 3.8 and 3.10 keep on holding, with the same proofs. The following lemma is analogous to Corollary 3.26, but it is not necessary to suppose that the orientation is proper.

Lemma 5.1 For $f: Y \rightarrow X$ a map of $h^{\bullet}$-oriented manifolds and $\theta \in H_{\mathrm{dR}}^{\bullet-1}\left(Y ; \mathfrak{h}_{\mathbb{R}}^{\bullet}\right.$, we have:

$$
f_{!}(a(\theta))=a\left(f_{!}(\operatorname{Td}(u) \cdot \theta)\right)
$$

This is equivalent to the fact that, for any $\alpha \in h^{\bullet}(X) \otimes_{\mathbb{Z}} \mathbb{R}^{3}$

$$
f_{!}(a(\operatorname{ch} \alpha))=a\left(\operatorname{ch}\left(f_{!} \alpha\right)\right)
$$

Proof Let us consider a differential Thom class $\hat{u}$ of $N_{\iota(Y)}\left(X \times \mathbb{R}^{N}\right)$ refining the orientation $u$ induced by the ones of $X$ and $Y$. We have:

$$
\begin{aligned}
f_{!}(a(\theta)) & =i_{*} \varphi_{*}\left(\hat{u} \cdot \pi^{*} a(\theta)\right)=i_{*} \varphi_{*}\left(a\left(\operatorname{dR}(R(\hat{u})) \cdot \pi^{*} \theta\right)=a\left(i_{*} \varphi_{*}\left(\operatorname{ch} u \cdot \pi^{*} \theta\right)\right)\right. \\
& =a\left(i_{*} \varphi_{*}\left(\operatorname{ch}^{(n)} u \cdot \pi^{*}(\operatorname{Td}(u) \cdot \theta)\right)=a\left(f_{!}(\operatorname{Td}(u) \cdot \theta)\right) .\right.
\end{aligned}
$$

Formula (24) follows from the Grothendieck-Riemann-Roch theorem.

Corollary 5.2 The Gysin map associated to $f: Y \rightarrow X$ induces a morphism of exact sequences of $\mathfrak{h}^{\bullet}$-modules:

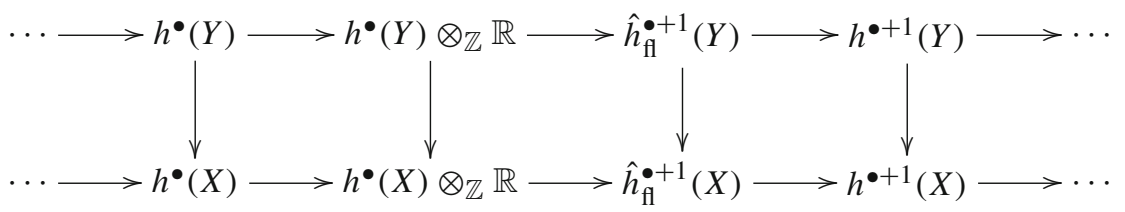

where the $\operatorname{map}^{\bullet}(X) \otimes_{\mathbb{Z}} \mathbb{R} \rightarrow \hat{h}_{\mathrm{fl}}^{\bullet+1}(X)$ is defined by $\alpha \mapsto a(\operatorname{ch} \alpha)$.

\footnotetext{
${ }^{3}$ In Eq. (24) we are considering the Chern character as defined on $h^{\bullet}(X) \otimes_{\mathbb{Z}} \mathbb{R}$, in which case it is an isomorphism. If we consider it as defined on $h^{\bullet}(X)$, then $a(\operatorname{ch} \alpha)=0$, and formula (24) implies coherently that $f_{!}(a(\operatorname{ch} \alpha))=0$.
} 


\subsection{Flat pairing}

We can now define a natural $\hat{\mathfrak{h}}_{\mathrm{fl}}^{\bullet}$-valued pairing on a manifold $X$ between $\hat{h}_{\mathrm{fl}}^{\bullet}$ and $h_{\bullet}$, that, in the case of singular differential cohomology, reduces to the holonomy of a flat Deligne cohomology class. If $\hat{h}_{\mathrm{fl}}^{\bullet} \simeq h^{\bullet}(\cdot ; \mathbb{R} / \mathbb{Z})$, such a pairing coincides with formula (22).

Definition 5.3 For $X$ a differential manifold, there is a natural pairing:

$$
\begin{gathered}
\xi_{\mathrm{fl}}^{n}: \hat{h}_{\mathrm{fl}}^{n}(X) \rightarrow \operatorname{Hom}_{\mathfrak{h}}\left(h_{n-\bullet}(X), \hat{\mathfrak{h}}_{\mathrm{fl}}^{\bullet}\right) \\
\hat{\alpha} \mapsto\left([M, u, \beta, f] \mapsto\left(p_{M}\right) !\left(\beta \cdot f^{*} \hat{\alpha}\right)\right) .
\end{gathered}
$$

We recall that $p_{M}$ is the unique map $p_{M}: M \rightarrow\{p t\}$ and the product $\beta \cdot f^{*} \hat{\alpha}$ is defined by (23). We have denoted by $\mathrm{Hom}_{\mathfrak{h}} \bullet$ the group of $\mathfrak{h}^{\bullet}$-invariant graded morphisms, which are by definition those morphisms such that:

$$
\xi_{\mathrm{fl}}^{n}(\hat{\alpha})([M, u, \beta, f] \cdot \gamma)=\xi_{\mathrm{fl}}^{n}(\hat{\alpha})([M, u, \beta, f]) \cdot \gamma
$$

In order to show that (25) is well-defined, i.e. that it does not depend on the representative $(M, u, \beta, f)$ of the homology class, and that formula (26) holds, we use an argument similar to the one used about (20).

Lemma 5.4 There is a morphism of complexes of $\mathfrak{h}^{\bullet}$-modules (the second one not being exact in general):

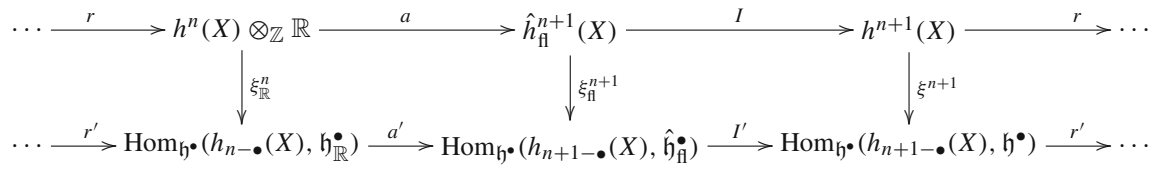

Proof We only have to prove the commutativity of the square under the map $a$. It easily follows from the fact that, for $\alpha \in h^{\bullet}(X) \otimes_{\mathbb{Z}} \mathbb{R}$ and $\beta \in h^{\bullet}(X)$ :

$$
a(\operatorname{ch} \alpha) \cdot \beta=a(\operatorname{ch}(\alpha \beta)) .
$$

That's because, for any differential refinement $\hat{\beta}$ of $\beta$, we have $a(\operatorname{ch} \alpha) \cdot \hat{\beta}=$ $a(\operatorname{ch} \alpha \cdot R(\hat{\beta}))=a(\operatorname{ch} \alpha \cdot \operatorname{ch} \beta)=a(\operatorname{ch}(\alpha \beta))$.

We call $\mathfrak{h}_{\mathbb{Z}}^{n}$ the image of the Chern character ch $: \mathfrak{h}^{n} \rightarrow H_{\mathrm{dR}}^{n}\left(p t ; \mathfrak{h}_{\mathbb{R}}^{\bullet}\right) \simeq \mathfrak{h}_{\mathbb{R}}^{n}$, which coindices with $\alpha \mapsto \alpha \otimes_{\mathbb{Z}} \mathbb{R}$.

Theorem 5.5 If $\mathfrak{h}^{\bullet}$ has no torsion, the pairing (25) is an isomorphism and $\hat{\mathfrak{h}}_{\mathrm{fl}}^{\bullet} \simeq$ $\mathfrak{h}_{\mathbb{R}}^{\bullet-1} / \mathfrak{h}_{\mathbb{Z}}^{\bullet-1}$. 
Proof The isomorphism $\hat{\mathfrak{h}}_{\mathrm{fl}}^{\bullet} \simeq \mathfrak{h}_{\mathbb{R}}^{\bullet-1} / \mathfrak{h}_{\mathbb{Z}}^{\bullet-1}$ easily follows from the long exact sequence $\cdots \rightarrow \mathfrak{h}^{\bullet-1} \rightarrow \mathfrak{h}_{\mathbb{R}}^{\bullet-1} \rightarrow \mathfrak{h}_{\mathrm{fl}}^{\bullet-1} \rightarrow \mathfrak{h}^{\bullet} \rightarrow \cdots$. In order to prove that (25) is an isomorphism, we consider the functor:

$$
k^{n}(X):=\operatorname{Hom}_{\mathfrak{h}}\left(h_{n-\bullet}(X), \mathfrak{h}_{\mathbb{R}}^{\bullet-1} / \mathfrak{h}_{\mathbb{Z}}^{\bullet-1}\right),
$$

defined on the same category on which $h^{\bullet}$ is defined. The functor $\hat{h}_{\mathrm{fl}}^{\bullet}$ is a cohomology theory [4, Sec. 7]. We show that also $k^{\bullet}$ is a cohomology theory. In fact, homotopy invariance follows from the one of $h_{\bullet}$. Moreover, since $\mathfrak{h}_{\mathbb{R}}^{\bullet-1} / \mathfrak{h}_{\mathbb{Z}}^{\bullet-1}$ is a division graded abelian group, the functor $\operatorname{Hom}_{\mathfrak{h}} \bullet\left(\cdot, \mathfrak{h}_{\mathbb{R}}^{\bullet-1} / \mathfrak{h}_{\mathbb{Z}}^{\bullet-1}\right)$ is exact, hence $k^{\bullet}$ has a natural Mayer-Vietoris sequence [4, sec. 7]. Finally, we show that $\xi_{\mathrm{fl}}^{\bullet}(p t): \hat{\mathfrak{h}}_{\mathrm{fl}}^{\bullet} \rightarrow k^{\bullet}(p t)$ is an isomorphism. Let us consider $1 \in \mathfrak{h}^{0}$ and the class $u_{1}:=\left[p t, u_{0}, 1, \mathrm{id}\right] \in h_{0}(p t)$. Any class belonging to $h_{n}(p t)$ is of the form $\left[p t, u_{0}, \gamma, \mathrm{id}\right]=u_{1} \cdot \gamma$. By $\mathfrak{h}^{\bullet}$-invariance, for any $\hat{\alpha} \in \mathfrak{h}_{\mathrm{fl}}^{n}$, we have $\xi_{\mathrm{fl}}^{n}(\hat{\alpha})\left(u_{1} \cdot \gamma\right)=\xi_{\mathrm{fl}}^{n}(\hat{\alpha})\left(u_{1}\right) \cdot \gamma$, hence the morphism $\xi_{\mathrm{fl}}^{n}(\hat{\alpha})$ is completely determined by the image of $u_{1}$, belonging to $\hat{\mathfrak{h}}_{\mathrm{fl}}^{n}$. It follows that $\xi_{\mathrm{fl}}^{n}: \hat{\mathfrak{h}}_{\mathrm{fl}}^{n} \rightarrow$ $k^{n}(p t)$ is an isomorphism.

\section{Generalized Cheeger-Simons characters}

We now describe a model of the homology groups $h_{\bullet}$, that involves a differential extension of the cycles, so that we will be able to define the generalized CheegerSimons characters.

Definition 6.1 On a smooth compact manifold $X$, we define:

- the group of $n$-precycles as the free abelian group generated by the quadruples $(M, \hat{u}, \hat{\alpha}, f)$, with:

- $(M, \hat{u})$ a smooth compact manifold without boundary with $\hat{h}^{\bullet}$-orientation $\hat{u}$, whose connected components $\left\{M_{i}\right\}$ have dimension $n+q_{i}$, with $q_{i}$ arbitrary;

- $\hat{\alpha} \in \hat{h}^{\bullet}(M)$, such that $\left.\hat{\alpha}\right|_{M_{i}} \in \hat{h}^{q_{i}}(M)$;

- $f: M \rightarrow X$ a smooth map;

- the group of $n$-cycles, denoted by $\hat{z}_{n}(X)$, as the quotient of the group of $n$-precycles by the free subgroup generated by elements of the form:

- $(M, \hat{u}, \hat{\alpha}+\hat{\beta}, f)-(M, \hat{u}, \hat{\alpha}, f)-(M, \hat{u}, \hat{\beta}, f)$;

- $(M, \hat{u}, \hat{\alpha}, f)-\left(M_{1},\left.\hat{u}\right|_{M_{1}},\left.\hat{\alpha}\right|_{M_{1}},\left.f\right|_{M_{1}}\right)-\left(M_{2},\left.\hat{u}\right|_{M_{2}},\left.\hat{\alpha}\right|_{M_{2}},\left.f\right|_{M_{2}}\right)$, for $M=$ $M_{1} \sqcup M_{2} ;$

- $\left(M, \hat{u}, \varphi_{!} \hat{\alpha}, f\right)-(N, \hat{v}, \hat{\alpha}, f \circ \varphi)$ for $\varphi: N \rightarrow M$ a submersion, oriented via the $2 \times 3$ principle;

- the group of $n$-boundaries, denoted by $\hat{b}_{n}(X)$, as the subgroup of $\hat{z}_{n}(X)$ generated by the cycles which are representable by a pre-cycle $(M, \hat{u}, \hat{\alpha}, f)$ such that there exists a quadruple $(W, \hat{U}, \hat{A}, F)$, where $W$ is a manifold and $M=\partial W, \hat{U}$ is an $\hat{h}^{\bullet}$ orientation of $W$ and $\left.\hat{U}\right|_{M}=\hat{u}, \hat{A} \in \hat{h}^{\bullet}(W)$ such that $\left.\hat{A}\right|_{M}=\hat{\alpha}$, and $F: W \rightarrow X$ is a smooth map satisfying $\left.F\right|_{M}=f$.

We define $h_{n}^{\prime}(X):=\hat{z}_{n}(X) / \hat{b}_{n}(X)$. 
Theorem 6.2 The natural group morphism:

$$
\begin{aligned}
& \Phi: h_{\bullet}^{\prime}(X) \rightarrow h_{\bullet}(X) \\
& {[(M, \hat{u}, \hat{\alpha}, f)] \rightarrow[(M, I(\hat{u}), I(\hat{\alpha}), f)]}
\end{aligned}
$$

is an isomorphism.

Proof We divide the proof in three steps.

Step 1 If $I(\hat{u})=I\left(\hat{u}^{\prime}\right)$ and $I(\hat{\alpha})=I\left(\hat{\alpha}^{\prime}\right)$, then $[(M, \hat{u}, \hat{\alpha}, f)]=\left[\left(M, \hat{u}^{\prime}, \hat{\alpha}^{\prime}, f\right)\right]$ in $h_{\bullet}^{\prime}(X)$. In fact, since $\hat{\alpha}^{\prime}=\hat{\alpha}+a(\rho)$, we consider on $I \times M$ the class $A=\pi_{I}^{*} \hat{\alpha}+$ $a\left(t \cdot \pi_{I}^{*} \rho\right)$, which links $\alpha$ to $\hat{\alpha}^{\prime}$. Moreover, we orient the projection $I \times M \rightarrow I$ in the following way: the embedding $M \hookrightarrow \mathbb{R}^{N}$ naturally determines an embedding $I \times M \hookrightarrow I \times \mathbb{R}^{N}$, with normal bundle $\pi_{I}^{*}\left(N_{M} \mathbb{R}^{N}\right)$; we put on such a bundle the Thom class $\hat{U}=\pi_{I}^{*} \hat{u}+a\left(t \cdot \pi_{I}^{*} \eta\right)$. Hence $\partial(I \times M, \hat{U}, \hat{A}, \mathrm{id} \times f)=(M, \hat{u}, \hat{\alpha}, f)-$ $\left(M, \hat{u}^{\prime}, \hat{\alpha}^{\prime}, f\right)$.

Step 2 Given two equivalent topological precycles $(M, u, \varphi ! \alpha, f) \simeq(N, v, \alpha, f \circ \varphi)$, any two differential refinements $\left(M, \hat{u}, \hat{\alpha}^{\prime}, f\right)\left(\right.$ with $\left.I\left(\hat{\alpha}^{\prime}\right)=\varphi ! \alpha\right)$ and $(N, \hat{v}, \hat{\alpha}, f \circ \varphi)$ are equivalent in $h_{\bullet}^{\prime}(X)$. In fact, by definition $\left[\left(M, \hat{u}, \varphi_{!} \hat{\alpha}, f\right)\right]=[(N, \hat{v}, \hat{\alpha}, f \circ \varphi)]$. By the first step, this implies that $\left[\left(M, \hat{u}, \hat{\alpha}^{\prime}, f\right)\right]=[(N, \hat{v}, \hat{\alpha}, f \circ \varphi)]$.

Step 3 The morphism $\Phi$ is clearly well-defined and surjective. Therefore, we only have to prove injectivity. Let us suppose that $\Phi[(M, \hat{u}, \hat{\alpha}, f)]=0$. Then $[(M, I(\hat{u}), I(\hat{\alpha}), f)]$ is equivalent, as a cocycle, to $[(N, v, \beta, g)]$ such that $(N, v$, $\beta, g)=\partial(W, V, B, G)$. This means that there exists a sequence of pre-cycles $\left(M_{i}, u_{i}, \alpha_{i}, f_{i}\right)$, for $i=0, \ldots, n$, such that $\left(M_{0}, u_{0}, \alpha_{0}, f_{0}\right)=(M, I(\hat{u}), I(\hat{\alpha}), f)$, $\left(M_{n}, u_{n}, \alpha_{n}, f_{n}\right)=(N, v, \beta, g)$ and such that there exists a submersion $\varphi_{i}: M_{i} \rightarrow$ $M_{i+1}$ or $\psi_{i}: M_{i+1} \rightarrow M_{i}$ such that $f_{i}=f_{i+1} \circ \varphi_{i}$ and $\alpha_{i+1}=\varphi_{!}\left(\alpha_{i}\right)$, or the analogue for $\psi_{i}$. We choose a differential refinement $\left(M_{i}, \hat{u}_{i}, \hat{\alpha}_{i}, f_{i}\right)$ for each $i$, such that for $i=$ 0 it coincides with $(M, \hat{u}, \hat{\alpha}, f)$. By the second step, we get that $\left[\left(M_{i}, \hat{u}_{i}, \hat{\alpha}_{i}, f_{i}\right)\right]=$ $\left[\left(M_{i+1}, \hat{u}_{i+1}, \hat{\alpha}_{i+1}, f_{i+1}\right)\right]$, hence $[(M, \hat{u}, \hat{\alpha}, f)]=[(N, \hat{v}, \hat{\beta}, g)]$. We now consider a differential refinement $(W, \hat{V}, \hat{B}, G)$ of $(W, V, B, G)$. By the step $1,[(N, \hat{v}, \hat{\beta}, g)]=$ $\left[\left(N,\left.\hat{V}\right|_{N},\left.\hat{B}\right|_{N}, g\right)\right]=0$.

Definition 6.3 A Cheeger-Simons differential $\hat{h}^{\bullet}$-character of degree $n$ on $X$ is a couple $\left(\chi_{n}, \omega_{n}\right)$, where:

$$
\chi_{n} \in \operatorname{Hom}_{\hat{\mathfrak{h}}^{\bullet}}\left(\hat{z}_{n-\bullet}(X), \hat{\mathfrak{h}}^{\bullet}\right) \quad \omega_{n} \in \Omega^{n}\left(X ; \mathfrak{h}_{\mathbb{R}}^{\bullet}\right)
$$

such that, if $(M, \hat{u}, \hat{\beta}, f)=\partial(W, \hat{U}, \hat{B}, F)$, then:

$$
\chi_{n}[M, \hat{u}, \hat{\beta}, f]=-a\left(\int_{W} \operatorname{Td}(W) \wedge R(\hat{B}) \wedge F^{*} \omega_{n}\right) .
$$

We have denoted by Hom $_{\hat{\mathfrak{h}}}$, the group of $\hat{\mathfrak{h}}^{\bullet}$-invariant graded morphisms, which are by definition those morphisms such that:

$$
\chi_{n}(\hat{\alpha})([M, \hat{u}, \hat{\beta}, f] \cdot \gamma)=\chi_{n}(\hat{\alpha})[M, \hat{u}, \hat{\beta}, f] \cdot \gamma
$$


We denote by $\breve{h}^{n}(X)$ the group of characters of degree $n$.

We briefly comment on formula (28). Let us suppose that $[M, \hat{u}, \hat{\beta}, f] \in \hat{z}_{n-k}(X)$ and that $M$ is connected. Then $\operatorname{dim}(M)=n-k+q$ and $\hat{\beta} \in \hat{h}^{q}(M)$, hence $\operatorname{dim}(W)=$ $n-k+q+1$ and $\hat{B} \in \hat{h}^{q}(M)$. Thus, in the r.h.s. of (28), we integrate on $W$ a $\mathfrak{h}_{\mathbb{R}}^{\bullet}$-valued form of degree $n+q+0$, hence we get a form on the point of degree $n+q-(n-k+q+1)=k-1$. Applying $a$, we get a class belonging to $\hat{\mathfrak{h}}^{k}$, as desired.

Theorem 6.4 There is a natural graded-group morphism:

$$
\begin{aligned}
& C S_{\hat{h}}^{\bullet}: \hat{h}^{\bullet}(X) \rightarrow \check{h}^{\bullet}(X) \\
& \hat{\alpha} \mapsto(\chi, R(\hat{\alpha})),
\end{aligned}
$$

where $\chi$ is defined, for $[M, \hat{u}, \hat{\beta}, f] \in \hat{z}_{n-k}(X)$, by:

$$
\chi[M, \hat{u}, \hat{\beta}, f]:=\left(p_{M}\right) !\left(\hat{\beta} \cdot f^{*} \hat{\alpha}\right) .
$$

Proof If we consider two representatives $(M, u, \varphi ! \beta, f)$ and $(N, v, \beta, f \circ \varphi)$ of the homology class, we have, thanks to Lemmas 3.24 and 3.27:

$$
\begin{aligned}
\chi[N, \hat{v}, \hat{\beta}, f \circ \varphi] & =\left(p_{N}\right) !\left(\hat{\beta} \cdot \varphi^{*} f^{*} \hat{\alpha}\right)=\left(p_{M}\right) ! \varphi !\left(\hat{\beta} \cdot \varphi^{*} f^{*} \hat{\alpha}\right) \\
& =\left(p_{M}\right) !\left(\varphi_{!} \hat{\beta} \cdot f^{*} \hat{\alpha}\right)=\chi[M, \hat{u}, \varphi ! \hat{\beta}, f] .
\end{aligned}
$$

Let us now suppose that $(M, \hat{u}, \hat{\beta}, f)=\partial(W, \hat{U}, \hat{B}, F)$. Then, for $\Phi$ defined as in 3.13, thanks to formula (9) one has:

$$
\left(p_{M}\right) !\left(\hat{\beta} \cdot f^{*} \hat{\alpha}\right)=\left.\left(\Phi_{!}\left(\hat{B} \cdot F^{*} \hat{\alpha}\right)\right)\right|_{\{0\}} .
$$

Since $\left.\left(\Phi_{!}\left(\hat{B} \cdot F^{*} \hat{\alpha}\right)\right)\right|_{\{1\}}=0$, because $\Phi^{-1}(1)=\emptyset$, from the homotopy formula (2) and Theorem 3.29 we have:

$$
\left(p_{M}\right) !\left(\hat{\beta} \cdot f^{*} \hat{\alpha}\right)=-a\left(\int_{I} R\left(\Phi_{!}\left(\hat{B} \cdot F^{*} \hat{\alpha}\right)\right)\right)=-a\left(\int_{W} \operatorname{Td}(W) \wedge R\left(\hat{B} \cdot F^{*} \hat{\alpha}\right)\right) .
$$

Hence:

$$
\chi[M, \hat{u}, \hat{\beta}, f]=-a\left(\int_{W} \operatorname{Td}(W) \wedge R(\hat{B}) \wedge F^{*} R(\hat{\alpha})\right) .
$$

This is exactly formula (28) for $\omega_{n}=R(\hat{\alpha})$. Finally:

$$
\begin{aligned}
\chi(\hat{\alpha})([M, \hat{u}, \hat{\beta}, f] \cdot \hat{\gamma}) & =\left(p_{M}\right) !\left(f^{*} \hat{\alpha} \cdot \hat{\beta} \cdot\left(p_{M}\right)^{*} \hat{\gamma}\right) \\
& =\left(p_{M}\right) !\left(f^{*} \hat{\alpha} \cdot \hat{\beta}\right) \cdot \hat{\gamma}=\chi(\hat{\alpha})[M, \hat{u}, \hat{\beta}, f] \cdot \hat{\gamma} .
\end{aligned}
$$


The proof of the following theorem is straightforward from the previous definition.

Theorem 6.5 If $\hat{\alpha}$ is flat, the value of the associated Cheeger-Simons character over $[M, \hat{u}, \hat{\beta}, f]$ coincides with the value of (25) on the corresponding homology class.

Considering the pairing (25), we remark that there is an embedding:

$$
j: \operatorname{Hom}_{\mathfrak{h}}\left(h_{n-\bullet}(X), \hat{\mathfrak{h}}_{\mathrm{fl}}^{\bullet}\right) \hookrightarrow \check{h}^{n}(X)
$$

In fact, a morphism $\varphi_{n} \in \operatorname{Hom}_{\mathfrak{h}}\left(h_{n-\bullet}(X), \hat{\mathfrak{h}}_{\mathfrak{f l}}^{\bullet}\right)$ determines a unique morphism $\chi_{n}: \hat{z}_{n-\bullet}(X) \rightarrow \hat{\mathfrak{h}}^{\bullet}$ defined by $\chi_{n}[M, \hat{u}, \hat{\beta}, f]:=\varphi_{n}[M, I(\hat{u}), I(\hat{\beta}), f]$, and we define $j\left(\varphi_{n}\right):=\left(\chi_{n}, 0\right)$. It follows from formula (28) that the image of $j$ is the subgroup of generalized Cheeger-Simons characters with vanishing curvature, which we call $h_{\mathrm{fl}}^{n}(X)$. Let us consider the embedding $i: \hat{h}_{\mathrm{fl}}^{\bullet}(X) \hookrightarrow \hat{h}^{\bullet}(X)$. The following diagram commutes:

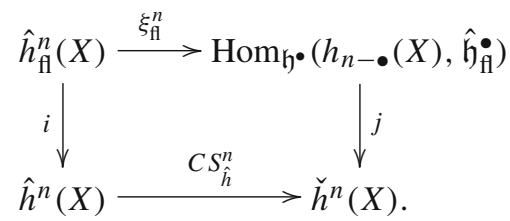

Therefore $i$ restricts to an embedding $i^{\prime}: \operatorname{Ker}\left(\xi_{\mathrm{ff}}^{n}\right) \hookrightarrow \operatorname{Ker}\left(C S_{\hat{h}}^{n}\right)$, and $j$ restricts to an embedding $j^{\prime}: \operatorname{Im}\left(\xi_{\mathrm{fl}}^{n}\right) \hookrightarrow \operatorname{Im}\left(C S_{\hat{h}}^{n}\right)$. Because of $j$ and $j^{\prime}$ we can construct a morphism $a: \operatorname{Coker}\left(\xi_{\mathrm{fl}}^{n}\right) \rightarrow \operatorname{Coker}\left(C S_{\hat{h}}^{n}\right)$. We now show that actually $i^{\prime}$ and $a$ are isomorphisms. In particular, if $\mathfrak{h}^{\bullet}$ has no torsion, $C S_{\hat{h}}^{n}$ is an isomorphism, because of Theorem 5.5.

Theorem 6.6 The following canonical isomorphisms hold:

$$
\operatorname{Ker}\left(\xi_{\mathrm{fl}}^{n}\right) \simeq \operatorname{Ker}\left(C S_{\hat{h}}^{n}\right), \quad \operatorname{Coker}\left(\xi_{\mathrm{fl}}^{n}\right) \simeq \operatorname{Coker}\left(C S_{\hat{h}}^{n}\right)
$$

Proof If $\hat{\alpha} \in \hat{h}^{n}(X)$ is not flat, then $C S_{\hat{h}}^{n}(\hat{\alpha}) \neq 0$, since $C S_{\hat{h}}^{n}(\hat{\alpha})=\left(\chi_{n}, R(\hat{\alpha})\right)$ and $R(\hat{\alpha}) \neq 0$. Hence $\operatorname{Ker}\left(C S_{\hat{h}}^{n}\right) \subset \operatorname{Ker}\left(\xi_{\mathrm{fl}}^{n}\right)$ and the equality follows. Moreover, $\check{h}_{\mathrm{fl}}^{n}(X) \cap \operatorname{Im}\left(C S_{\hat{h}}^{n}\right)=\operatorname{Im}\left(\xi_{\mathrm{fl}}^{n}\right)$, hence $a: \operatorname{Coker}\left(\xi_{\mathrm{fl}}^{n}\right) \rightarrow \operatorname{Coker}\left(C S_{\hat{h}}^{n}\right)$ is an embedding. If $\left(\chi_{n}, \omega_{n}\right) \in \breve{h}^{n}(X)$, we consider a class $\hat{\alpha} \in \hat{h}^{n}(X)$ such that $R(\hat{\alpha})=\omega_{n}$, and we call $\left(\chi_{n}^{\prime}, \omega_{n}\right):=C S_{\hat{h}}(\hat{\alpha})$. Then $\left(\chi_{n}^{\prime}-\chi_{n}, 0\right) \in \breve{h}_{\mathrm{fl}}^{n}(X)$, and, in $\operatorname{Coker}\left(C S_{\hat{h}}^{n}\right)$, one has $\left[\left(\chi_{n}, \omega_{n}\right)\right]=\left[\left(\chi_{n}^{\prime}-\chi_{n}, 0\right)\right] \in \operatorname{Im} a$. Therefore $a$ is also surjective.

\subsection{Singular cohomology and K-theory}

In the case of singular cohomology, we have $\hat{\mathfrak{h}}^{0} \simeq \mathbb{Z}, \hat{\mathfrak{h}}^{1} \simeq \mathbb{R} / \mathbb{Z}$ and $\hat{\mathfrak{h}}^{n}=0$ for $n \neq 0,1$. Moreover, $\hat{\mathfrak{h}}_{\mathrm{fl}}^{1} \simeq \mathbb{R} / \mathbb{Z}$ and $\hat{\mathfrak{h}}_{\mathrm{fl}}^{n}=0$ for $n \neq 1$. Thus, a flat differential class 
$\hat{\alpha} \in \hat{H}_{\mathrm{fl}}^{n}(X)$ defines a differential character $\chi_{n}: H_{n-1}(X) \rightarrow \mathbb{R} / \mathbb{Z}$. Such a character corresponds (up to the exponential) to the holonomy of the corresponding smooth Deligne cohomology class, for which there is an explicit formula [7]. Actually, the holonomy is defined on singular cycles, with no need of differential refinement. Thus, there is a canonical isomorphism between the group of Cheeger-Simons characters defined on singular cycles (in the usual sense), and the group of Cheeger-Simons characters defined on differential cycles (as defined in the present paper), both groups being canonically isomorphic to $\hat{H}^{n}(X)$. We can explicitly describe this isomorphism for a large class of cycles. In fact, let us consider an $(n-1)$-cycle $[(M, \hat{u}, 1, f)$, for $\hat{u}$ any differential orientation refining the topological one. Then, for $\alpha \in \hat{H}^{n}(X), f^{*} \hat{\alpha}$ is flat and with trivial first Chern class for dimensional reasons, therefore $f^{*} \hat{\alpha}=[h] \in$ $H^{n}(M ; \mathbb{R}) / H^{n}(M ; \mathbb{Z})$ and the holonomy coincides with the exponential of $h([M])$, $[M]$ being the fundamental class of $M$.

Finally, we consider the case of complex K-theory. We call $\mathfrak{k}^{\bullet}$ the $K$-theory ring of the point. In this case $I$ provides a canonical isomorphism:

$$
\hat{\mathfrak{k}}^{2 n} \simeq \mathfrak{k}^{2 n} \quad \forall n \in \mathbb{Z},
$$

because $\Omega^{2 n-1}\left(p t, \mathfrak{k}_{\mathbb{R}}^{\bullet}\right) \simeq \mathfrak{k}_{\mathbb{R}}^{2 n-1}=0$. Moreover, let us consider the class $\gamma_{0} \in \mathfrak{k}^{-2}$, corresponding to the dual of the tautological line bundle of $\mathbb{P}^{1}(\mathbb{C})$. Then Bott periodicity $K^{n}(X) \simeq K^{n-2}(X)$ is given by $\alpha \mapsto \alpha \cdot \gamma_{0}$. Such a periodicity can be extended to differential K-theory. In fact, because of (32), there is a unique differential extension $\hat{\gamma}_{0}$, hence the map $\hat{\alpha} \mapsto \hat{\alpha} \cdot \hat{\gamma}_{0}$ is an isomorphism. It follows that $\hat{\mathfrak{k}}^{2 n} \simeq \hat{\mathfrak{k}}^{0} \simeq \mathbb{Z}$. Moreover, $\hat{\mathfrak{k}}^{2 n+1} \simeq \hat{\mathfrak{k}}^{1} \simeq \mathbb{R} / \mathbb{Z}$, because the elements of $\hat{\mathfrak{k}}^{1}$ are of the form $a(\theta)$, with $\theta \in H_{\mathrm{dR}}^{0}\left(p t ; \mathfrak{k}_{\mathbb{R}}^{\bullet}\right) \simeq \mathfrak{k}_{\mathbb{R}}^{0} \simeq \mathbb{R}$, and $a(\theta)=0$ if and only if $\theta \in \mathbb{Z}$. The periodicity can be extended to differential cycles, via the isomorphism:

$$
\begin{aligned}
& B: \hat{z}_{n}(X) \rightarrow \hat{z}_{n-2}(X) \\
& {[M, \hat{u}, \hat{\beta}, f] \mapsto\left[M, \hat{u}, \hat{\beta} \cdot \hat{\gamma}_{0}, f\right] .}
\end{aligned}
$$

Therefore, a generalized Cheeger-Simons character, as defined by formula (27), is uniquely determined by its restriction to $\hat{z}_{n-1}$, because of formula (29). The same holds for the pairing (25) (see [13] for an analytic description of the pairing). It follows from Theorem 5.5 or from the universal coefficient theorem for K-theory [18, formula3.1], that (25) and (30) are isomorphisms. Therefore, in the case of K-theory, the pairing (25) and Theorem 6.4 can be summarized and enriched as follows.

Definition 6.7 A Cheeger-Simons differential $\hat{K}^{\bullet}$-character of degree $n$ on $X$ is a couple $\left(\chi_{n}, \omega_{n}\right)$, where:

$$
\chi_{n}: \hat{z}_{n-1}(X) \rightarrow \mathbb{R} / \mathbb{Z} \quad \omega_{n} \in \Omega^{n}\left(X ; \mathfrak{k}_{\mathbb{R}}^{\bullet}\right)
$$

such that, if $(M, \hat{u}, \hat{\beta}, f)=\partial(W, \hat{U}, \hat{B}, F)$, then $\chi_{n}[(M, \hat{u}, \hat{\beta}, f)] \equiv_{\mathbb{Z}} \int_{W} \operatorname{Td}(W) \wedge$ $R(\hat{B}) \wedge F^{*} \omega_{n}$. We denote by $\check{K}^{n}(X)$ the group of characters of degree $n$. 
Theorem 6.8 There is a natural group isomorphism:

$$
\begin{gathered}
C S_{K}^{n}: \hat{K}^{n}(X) \rightarrow \check{K}^{n}(X) \\
\hat{\alpha} \rightarrow(\chi, R(\hat{\alpha})),
\end{gathered}
$$

where $\chi$ is defined, for $[(M, \hat{u}, \hat{\beta}, f)] \in \hat{z}_{n-1}(X)$, by $\chi[(M, \hat{u}, \hat{\beta}, f)]:=\left(p_{M}\right)_{!}(\hat{\beta}$. $\left.f^{*} \hat{\alpha}\right)$. Restricting to flat classes, we get an isomorphism:

$$
\xi_{\mathrm{fl}}^{n}: \hat{K}_{\mathrm{fl}}^{n}(X) \rightarrow \operatorname{Hom}\left(K_{n-1}(X), \mathbb{R} / \mathbb{Z}\right) .
$$

This construction is equivalent to the one considered in [2]. In fact, let us call $\mathcal{C}_{\bullet}(X)$ the groups of $K$-cycles, as defined in [2]. An element of $\mathcal{C}_{\bullet}(X)$ is represented by a triple $(M,(E, h, \nabla), \phi)$, where $M$ is a compact manifold with fixed metric and spin $^{c}$-structure, $E \rightarrow X$ is a vector bundle with Hermitian metric $h$ and compatible connection $\nabla$, and $\phi: M \rightarrow X$ is a smooth function. ${ }^{4}$ Using the Freed-Lott model [5, def. 2.15, p. 8], $(E, h, \nabla)$ defines a differential $K$-theory class $\hat{\alpha}$. Moreover, the fixed metric and $\operatorname{spin}^{c}$-structure define a Thom class of the tangent bundle of $M[12$, theorem C.12, p. 388], hence, by the $2 \times 3$ principle, define an orientation of the stable normal bundle of $M$. The differential refinement of the Thom class is provided by the canonical metric and connection on the spinor bundle, again using the Freed-Lott model. In this way we get a differential $K$-cycle as defined in the present paper. Since the vector bundle modification, considered in [2], is a particular case of the Gysin map, we get a morphism $\rho: \mathcal{C}_{\bullet}(X) \rightarrow \hat{z}_{\bullet}(X)$. Finally, let us call $\check{\mathcal{K}}^{\bullet}(X)$ the group of differential $K$-characters, as defined in [2]. We define $\nu^{\bullet}: \check{K}^{\bullet}(X) \rightarrow \check{\mathcal{K}}^{\bullet}(X)$ as $\nu(\chi)(A):=\chi(\rho(A))$ and the identity on the curvature. Both the groups $\check{K}^{\bullet}(X)$ and $\check{\mathcal{K}}^{\bullet}(X)$ fit in the exact sequence $0 \rightarrow K^{\bullet}(X ; \mathbb{R} / \mathbb{Z}) \rightarrow \check{K}^{\bullet}(X) \rightarrow \Omega_{0}^{\bullet+1}(X) \rightarrow 0[2$, Theorem 1, p. 431], and the morphism $v^{\bullet}$ commutes with the identities of $K^{\bullet}(X ; \mathbb{R} / \mathbb{Z})$ and $\Omega_{0}^{\bullet+1}(X)$. Because of the five lemma, $v^{\bullet}$ is an isomorphism.

\section{References}

1. Adams, J.F., Stable homotopy and generalized homology. University of Chicago Press, Chicago, London (1974)

2. Benameur, M.-T., Maghfoul, M.: Differential characters in K-theory. Differ. Geom. Appl. 24, 417-432 (2006)

3. Bunke, U.: Differential Cohomology, Course Note. arXiv:1208.3961

4. Bunke, U., Schick, T.: Uniqueness of smooth extensions of generalized cohomology theories. J. Topol. 3(1), 110-156 (2010). arXiv:0901.4423

5. Freed, D.S., Lott, J.: An index theorem in differential K-theory. Geom. Topol. 14, 90366 (2010). arXiv:0907.3508

6. Freed, D., Moore, G., Segal, G.: The uncertainty of fluxes. Commun. Math. Phys. 271, 247-274 (2007). arXiv:hep-th/0605198

7. Gomi, K., Terashima, Y.: Higher dimensional parallel transport. Math. Res. Lett. 8, 2533 (2001)

8. Hopkins, M.J., Singer, I.M.: Quadratic functions in geometry, topology, and M-theory. J. Differ. Geom. 70, 329-452 (2005). arXiv:math/0211216

\footnotetext{
${ }^{4}$ In [2] the function $\phi$ is only supposed to be continuous, but the result is the same by smooth approximation theorem.
} 
9. Jakob, A.: A bordism-type description of homology. Manuscripta Math. 96, 67-80 (1998)

10. Karoubi, M.: K-Theory: An Introduction. Springer, Berlin (1978)

11. Kosinski, A.: Differential Manifolds. Academic Press, Inc., New York (1993)

12. Lawson, H.B., Michelsohn, M.L.: Spin Geometry. Princeton University Press, Princeton (1998)

13. Lott, J.: $\mathbb{R} / \mathbb{Z}$ index theory. Commun. Anal. Geom. 2(2), 279-311 (1994)

14. Rudyak, Y.B.: On thom spectra, orientability and cobordism. In: Springer Monographs in Mathematics. Springer, Berlin

15. Switzer, R.M.: Algebraic Topology. Springer, Berlin (1975)

16. Upmeier, M.: Algebraic structure and integration maps in cocycle models for differential cohomology. Algebr. Geom. Topol. 15, 65-83 (2015). arXiv:1112.4173

17. Whitehead, G.W.: Generalized homology theories. In: Transactions of the American Mathematical Society, vol. 102, No. 2 (Feb., 1962), pp. 227-283

18. Yosimura, Z.: Universal coefficient sequences for cohomology theories of CW-spectra. Osaka J. Math. 12, 305-323 (1975) 